
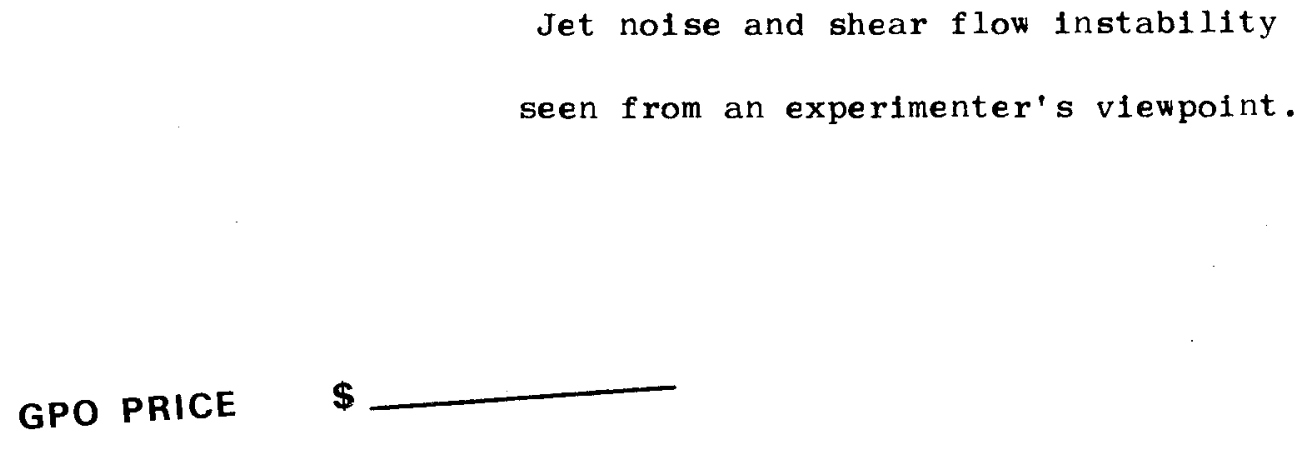

CSFTI PRICE(S) \$_ by

Hard copy $(H C) \frac{3.00}{.69}$

ff 603 July 65

Erik Mollo-Christensen
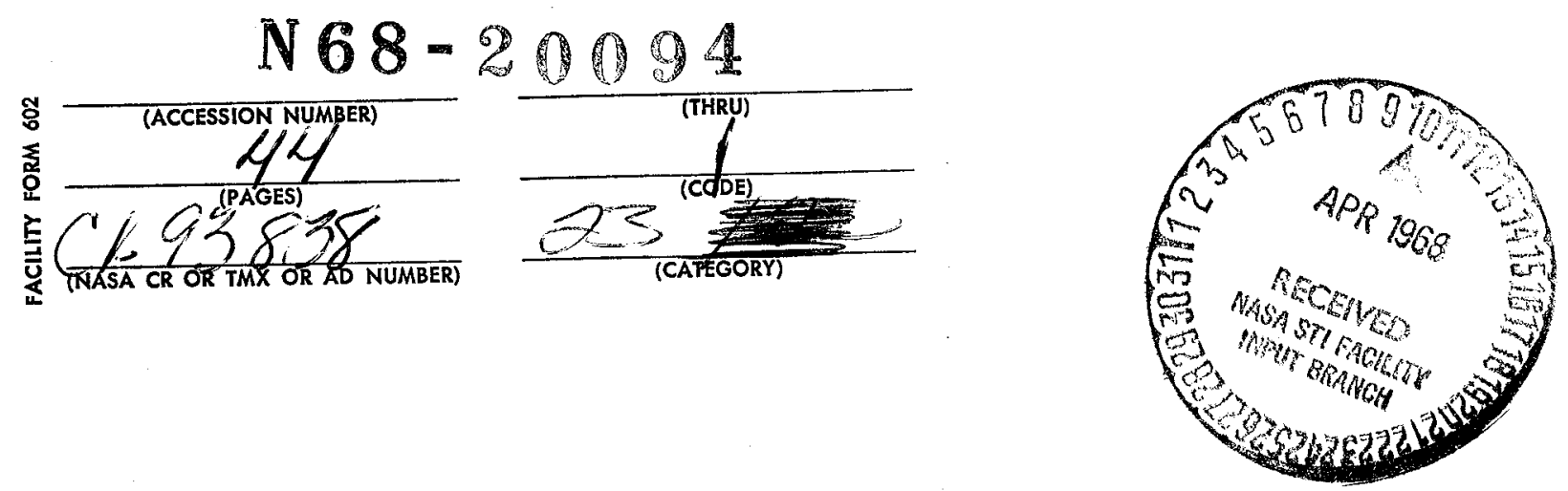


\begin{abstract}
Discussion of similarity laws for jet noise as suggested by experiments. Construction of a model line emitter. Near field pressure covariances and spectra. Phase coherence of the near pressure field, non-linear couplings in the process of generation of turbulence.
\end{abstract}

ACKNOWLEDGEMENTS

This work was supported by NASA under Grant NsG-31-60

and Grant NsG-496. 
In this talk I shall report on some experiments we have done on jet nolse; I will attempt to sketch the status of our knowledge and our ignorance in this field. Incidentally, I will expose you to a point of view, and show you some of the interesting but difficult phenomena which occur in turbulent jets.

The father of the theory of jet noise is of course Lighthill, who sugested the following useful approximation to the far field pressure fluctuations caused by a region of turbulence imbedded in quiescent air (Eq. 1).

$$
\begin{aligned}
& p(\vec{r}, t) \simeq \frac{r_{i} r_{j}}{4 \pi a^{2}|\vec{r}|^{3}} \iiint_{V_{\vec{x}}} \frac{\partial^{2}}{\partial t^{3}}\left(g u_{i} u_{j}\right) \\
& r \text { is the distance from the emitting region; } u_{i} \text { and } u_{j} \text { are velocity }
\end{aligned}
$$
fluctuations, $p$ is pressure and $a$ the speed of sound. The integral is evaluated at retarded time, $|\vec{r}-\vec{x}| / a$ being the time it takes a signal to go from $\vec{x}$ to $\vec{r}$.

This formula has produced many useful similarity relations, it has been a guide post for all subsequent work on jet nolse.

Ribner has suggested a modification of Eq. (1), namely to express the integrand in terms of pressure fluctuations in the turbulent region

$$
p(\vec{r}, t) \simeq \frac{r_{i} r_{j}}{4 \pi a^{2}|\vec{r}|^{3}} \iiint_{V_{\vec{x}}}\left(\frac{\partial^{2} p}{\partial t^{2}}\right)_{\left[\vec{x}, t-\frac{|\vec{x}-\vec{r}|}{u}\right]} d V^{\prime}
$$

and we shall interpret our results in a similar way. 
Since the turbulent field of a jet is random, we have to be content by a statistical description. Whether one considers the use of statistics a confession of ignorance or an ability to rise above trivial detail does not matter; one still finds that one must remain content with descriptions of the lowest orders of statistical measures.

These are the mean square fluctuation, the time correlation or its Fourier transform, the power spectral density. The far field time corm relation of pressure is, in terms of Lighthill's approximation,:

$$
\begin{aligned}
& r \text { r } r \\
& \langle p(\vec{r}, t) p(\vec{k}, t+\tau)\rangle=R_{p p}(\vec{k}, t)
\end{aligned}
$$

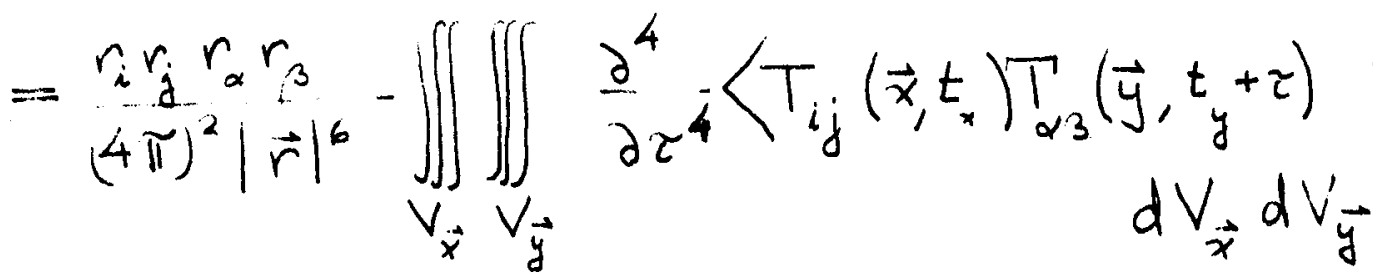

where $T_{i j}=g u_{i} u_{j} \quad, t_{x}$ and $t_{y}$ are the retarded times appropriate to the locations $\vec{x}$ and $\vec{y}$, and \langle\rangle denotes an ensemble average.

An experimenter looking at Eq. (3) is not going to be very happy. It tells him that the pressure time correlation in the far field, which he can measure using a single microphone and some elecironic gadgetry, can also be found by measuring quadruple space-time correlations of the velocity fluctuations for all pairs of points $\vec{x}$ and $\vec{y}$ within the jet, and then evaluate the sextuple integral. This is a very ambitious undertaking, and is one reason why it has never been done. Besides just the problems of complexity of such measurements, it is difficult to use 
hot wires at high subsonic Machnumbers. The hot wires give plenty of output, but it is very difficult to distinguish between velocity and temperature fluctuations.

When we started our measurements, we realized that the theory of ae rodynamic nolse could never be checked in detail. Furthermore, acoustics is much easier to understand than turbulence. We therefore decided to stress measurements near and in the fets, hoping to discern some of the simpler features of the turbulent field. We also did measure for field pressures, and intended to see if we could not connect the two sets of ooservations somehow, using the equations of sound propagation.

\section{Experimental set-up.}

Fig. 1 shows the experimental set-up. It is included because it was so expensive--expensive mostly in terms of the extended effort required to eliminate parasitic sources of turbulence and notse. Our criterion was that the far field spectra should not change when we changed jet velocity, diameter and other parameters, and which decreased in. amplitude as $1 / r$ when the microphone was moved. When one manages to modify the apparatus to eliminate the more obvious parasitic signals, one is able to discern others which were hidden before; they again mask the st 111 smaller ones.

After extensive modifications over a long period of time, we finally reached a stage where narrow band spectra of sound and velocity fluctuations only depended upon jet parameters, and not upon any others, such as pipe vibrations and upstream velocity and temperature fluctuations. 


\section{Results}

The first question one has to ask is how well do small jets simulate larger ones. Fig. 2 shows the power spectral density of sound emitted from jets from different nozzles. The diameter and nozzle lengths vary. The point in the far field chosen is the worst one for similarity. The small, short nozzle has a laminar boundary layer on the nozzle wall at the exit and the others have turbulent boundary layers.

We also tried one nozzle with vortex generators on the nozzle wall several diameters upstream of the exit plane.

It can be seen that when the wall boundary layer is turbulent, Reynolds number ceases to be an important parameter, and all the spectra are similar.

Fig. 3 shows the mean Machnumber profiles, and the hypothesis that shear layer thickness grows linearly with distance from the nozzle exit seens valid.

We were then ready to start poking around with microphones in the near field of the jets, and Fig. 4 shows an installation of two microphones at opposite sides of a jet. Among the quantities we measured were space - time correlations of pressure fluctuations in the immediate vicinity of the jets.

A simplified block diagram of the data-recording system is shown in Fig. 5 .

Using a magnetic tape recorder to obtain a relative time delay between the signals from two microphones, multiplying the signal and 
averaging the product, we obtained their crossmcorrelation.

Plotting correlation versus time delay on an $x-y$ plotter, the correlation was obtained.

Fig. 6 shows examples of correlation curves obtained this way. Before a run, we recorded the autocorrelation of a $20 \mathrm{kc}$ sinusoidal signal. This is a cosine curve with period 50 microseconds in delay time $\tau$

We then recorded the autocorrelation of white, or rather grey nolse, which has a sharp maximum at zero time delay. This provided the origin and scale in correlation time.

Fig. 6 shows autocorrelations of near field pressure for two Machnumbers, the delay time has been scale with jet exit velocity. The similarity is apparent.

Using two microphones just outside the jet, separated by a distance $\Delta x=\xi$ in the axial direction, we obtained the correlation coefficient curves shown in Fig. 7 for two exit Machnumbers, using a scaled delay time. The similarity is evident.

It is also interesting to note that the correlation phase takes more time to reach a certain $\xi$ than does correlation amplitude.

one may say that the correlation phase speed is less than the "group" speed.

If one plots phase displacement versus delay time, one obtains the results shown in Fig. 8 which shows that the phase speed increases with distance from the nozzle exit. This is reasonable, since an increasing part of the pressure signal is sound, and moves at the speed of sound with respect to the medium. 
$-6-$

Measuring the pressure correlation coefficient across the jet, we obtained the results shown in Fig. 9 .

Beyond the point where the boundary layer on the nozzle wall is turbulent, which is at Machnumber 0.6 for this particular jet, the corm relations show a similar behavior with distance from the nozzle exit plane.

Fig. 10 shows how the root mean square pressure varies along the jet. It increases with distance up to one diameter away from the exit plane and then decays.

4. A simple model emitter.

The data suggest that one may perhaps represent the fluctuating pressure field in terms of rather simple functions.

For example, one may consider the jet as a semi-infinite line emitter, a semi-infinite antenna for sound, if you like.

A possible distribution of sound sources of a certain wave-number might be:

$$
Q_{n}(x, t)=\frac{\partial^{n}}{\partial(-H)^{n}} \exp \{-H x+i k(x-V t)\} d A_{n}(k) \quad(4)
$$

where $\mathcal{H}$ is a decay parameter, $k$ the wave-number, $V$ a phase speed and $d A(k)$ a random function of wave-number.

$$
\text { The set of functions } Q_{n}(x, t) \text { are related to the Laguerre }
$$
polynomials in $X$, a nd thus format complete set on the interval $[0, \infty)$

It is easy to compute the far field from such an emitter. The corresponding far field pressure at $r, \vartheta$ and $t$ is: 


$$
p_{n}\left(r_{r} \vartheta, t\right)=\frac{-e^{i k M(r-a t)}}{4 \pi r} \frac{\partial^{n}}{\partial(-\phi)^{n}}\left[\frac{d A_{n}(k)}{-\ell+i k(1-M \cos \theta)}\right]
$$

(5)

where $M=V^{\prime} / a$.

Fig. 11 shows how the source density $Q_{1}$ varies along $x$.

By differentiating this expression with respect to the space varabies, we can find the fields for dipoles and quadrupole with density distributions $Q_{M}$ along the $x$-axis.

The choice of this distribution illustrates an important point I would like you to note. If you think you know some of the features of a random function, for goodness sake put it in, and don't just use the general form of the Fourier-stieltjes integral, but pull out of the Fourier amplitude $d A_{M}(\ell)$ as much as you can, such as the fact that the disturbances do move, that their amplitude changes with $X$ and disappears at infinity etc. This leaves only our real ignorance hidden in. the statist1cs.

From Eq. 5 we can compute the far field pressure correlation from a quadruple distribution with density $Q_{1}$ :

Set:

$$
C_{1}=\rho U^{2} \int_{-\infty}^{+\infty} x \exp \{-j x-i k(x-V t)\} d A(k)
$$

We obtain:

$$
\langle p(r, \theta, t) p(r, t, t+t)\rangle
$$

$$
=\frac{\rho^{2} U^{4} F(\theta)}{(4 \pi)^{2} r^{2}} \int_{-\infty}^{+\infty} \frac{M^{4} k^{4} \phi(k) e^{-i k M a \tau} d k}{\left[H^{2}+k^{2}(1-M \cos \theta)^{2}\right]^{2}} \text {. }
$$


$-8-$

or, with a change in variable, to reflect the fact that waves travelling along the emitter appear foreshortened when projected on $r$ :

$$
\begin{aligned}
& \langle p(r, \theta, t) p(r, \theta, t+\tau)\rangle= \\
& =\frac{E(\theta) g^{2} U^{4} M^{4}}{(4 \pi)^{2} r^{2}} \int_{-\infty}^{+\infty} \frac{\gamma^{4} e^{-i \frac{\gamma M a}{1-M \cos \theta} \tau} \phi(\gamma /(1-M \cos \theta)) d \gamma}{\left(H^{2}+\gamma^{2}\right)^{2}(1-M \cos \theta)^{5}}
\end{aligned}
$$

$\phi(k)$ is the wavenumber spectral density of the emitter:

$$
\phi(k) d k=\int_{-\infty}^{+\infty}\left\langle d A\left(k_{1}\right) d A^{*}\left(k-k_{1}\right)\right\rangle d k_{1}
$$

where the angular brackets denote an ensemble average.

$F(\theta)$ is a function which depends upon the directions of the qiladrupoles, assuming the statistical measures of quadrupole direction to be axisymmetrical.

This dependence upon $M$ and $\theta$ has been obtained previously by Williams, namely that the far field signal is proportional to:

$$
\rho^{2} \cup^{4} M^{4} /(1-M \cos \theta)^{5}
$$

$\gamma_{\text {is }}$ the new wave number variable, chosen so as to give the proper 


\section{$-9-$}

Doppler sealing between emitter wave number and wave number in the far field.

Our model emitter thus behaves according to established tradition. We may next ask how much of each kind of quadrupoles should the model emitter contain, what is the phase velocity of each wave number component, and in general examine the near field in more detail in the hope that we can calculate the far field.

\section{Pressure correlations for different frequency bands.}

Let us first look at our results for space-time correlations of individual frequency bands. They were obtained using bandpass filters for each microphone signal before putting the signals into the correlator, as shown in Fig. 12 .

A typical set of results are shown in Fig. 13. The autocorrelation shown in the top of the figure is more a characteristic of the bandpass filtar than of the pressure signal so we have to avold attributing any deeper significance to it.

The space-time cross-correlations, however, indicate that we may find phase speed and correlation amplitude variation for each frequency band.

The correlation amplitude variation is shown in Fig. 14q, showing that correlations do first increase and then decay, but differently for each frequency band. The higher the frequency, the further upstream is its maximum correlation, but the maxima do occur at finite $\Delta x$, rather than at zero $\Delta x$. The highest frequencies have their maximum correlation further upstream than shown in the figure. 
The phase displacements versus delay time for several frequencies are shown in Fig. 14ab It is difficult to find a systematic variation with either frequency, Machnumber or distance from the jet exit $x$. The phase speed seems to increase with distance; this is reasonable since one should expect that more and more of the correlated part of the signal is sound as the microphones are moved further apart.

Next we take a look at the pressure correlations across the jet, in order to find estimates of the relative content of axisymmetric, elliptical and doubly bulged pressure field around the jet (Fig. 15).

This shows a crude breakdown of the circumferential correlation field into three terms:

$$
\begin{aligned}
& \langle f(x, \alpha, t) f(x, \alpha+\Delta \alpha, t)\rangle= \\
& =A(x,+B(x) \cos (\Delta \alpha)+C(x) \cos 2(\Delta \alpha)
\end{aligned}
$$

A $x$ is the fraction which is axisymmetric, [ T the fraction of "dipole" structure lateral to the jet axis; and $C_{(} x_{j}$ is the fraction with "quadrupole" structure normal to the jet axis. Knowing the phase speeds, the maxima and decay parameters for different frequency components, and the power spectral density of pressure fluctuations at the jet, we should be able to compute the far field power spectrim. The power spectral density of the near field is shown in Fig. 16, typical far field spectra in Fig. 17. 
It is a pity it doesn't work. The reason is that emitters of different type have different power spectral densities.

Just looking at the far field spectra we see that this must be the case.

The persistence of high frequency emission at larger values of $\theta$ indicates that the jet must contain high frequency emitters which emit predominantly in a direction almost normal to the jet, while the low frequency emitters emit in directions closer to the jet axis.

This directionality is also quite apparent if one compares the total rms pressure in the far field (Fig. 16) with the rms pressure in low (Fig. 19) and high (Fig. 20) frequency bands.

We measured space-time correlations of pressure fluctuations between different frequency bands, using the set-up shown in Fig. 12, just that the bandpass filters were tuned to different frequencies.

Fig. 2 shows some sample results. In the left half of Fig. 2 , the upstream microphone output was tuned to pass the band 8 to $9 \mathrm{kc}$, the downstream microphone output to 6 to $7 \mathrm{kc}$. The space time correlation remains significantly different even for a microphone separation of $1.5 \mathrm{D}$.

This might be rather lupsetting to those who are accustomed to thinking of the world in terms of linear systems.

What the data are telling us is that turbulence comes in packages, containing components of all frequencies, and that different frequency components preserve their phase relationships over a few jet diameters. One of my students, Dr. Browand, investigated the instability processes in a two-dimensional free shear layer, measuring velocity fluctuations in the layer. The power spectral density of velocity fluctuations 
in the early shear layer was measured using a loud speaker just outside the wind tunnel to trigger the instability. (Fig. 22) The spectrum of forced instability oscillations show sharp distinct peaks at the forcing frequency $\omega_{0}$ and at $2 \omega_{0}$, and also at the subharmonic frequency $\omega / 2$ and its harmonics $3 \omega_{0 / 2}$ and $5 \omega_{0,2}$. The forcing apparently fixes the relative phase of the frequency components caused by nonlinear interactions.

Removing the forcing, one obtains a smoother spectrum, as shown. The smoothing most probably results from phase randomization of the fundamental instability. Therefore, although the velocity signal now is random, one should expect to see intermittently a rather regular spatial structure in the shear layer.

Using some imagination, one may also discern such a regular structure in a shadow picture of a jet (Fig. 23).

A further piece of supporting evidence is found by looking at a space-time correlation of pressure near a low speed jet (F1g. 24) where, for small correlation distances, one frequency seems to dominate the cross-correlation, while at larger separation distances, a subharmonic oscillation is dominant.

Whether the process can be described as "the tail wagging the dog", 1.e. that the early jet responds to the pressure field associated with fluctuations further downstream, or the process is one of parametric amplification, as described by Kelly, is interesting, and deserves to be pursued further. 


$$
-13-
$$

\section{Conclusions}

There are obviously problems left to be solved in the field of jet noise. They involve some fundamental questions about the statistical structure of shear flow turbulence. It is suggested that turbulence, at least as far as some of the lower order statistical measures are concerned, may be more regular than we think it is, if one only could find a new way of looking at it. 
$-14-$

ACKNOWLEDGEMENTS

This work was supported by NASA under Grant NsG-31-60 and Grant Nst:-496. 
REFERENCES

Lighthill, M. J.: Sound generated aerodynamically. The Bakerian Lecture, 1961. RAE Tech. Mem. No. DIR 8 (1962).

Ribner, H. S.: The generation of sound by turbulent jets. Advances in Applied Mechanics, Vol. 8, Academic Press, New York.

Williams, J. E. F.: The noise from turbulence convected at high speed. Phil. Trans. Roy. Soc., London, Series A, 255, 469-503 (1963)

Browand, F. K.: An experimental investigation of an incompressible, separated shear layer. M.I.T. Ph.D. Thesis, 1965.

Kelly, R. E.: The stability of unsteady Kelvin-Helmholz flow. Jour. Fluid Mech., 22, 547-560 (1965). 


\section{LIST OF FIGURES}

Fig. 1 Experimental Arrangements.

Fig. 2 Spectra of far field pressure fluctuations from different jet nozzles.

Fig. 3 Machnumber profiles for jet from $\frac{1}{2}$ in diameter nozzle.

Fig. 4 Installation of microphones near a jet.

Fig. 5 Block diagram of data processing system for obtaining space-time correlations.

Fig. 6 Sample autocorrelation of pressure near a jet.

Fig. 7 Space-time correlations of pressures near a jet.

Fig. 8 Phase speed of pressure fluctuations near jets varsus microphone separation $\Delta x$.

Fig. 9 Pressure correlations across a jet diameter versus distance from exit.

Fig. 10 Variation of rms pressure fluctuation with downstream distance.

Fig. 11 sketch of variation of emitter density.

Fig. 12 Block diagram of cross correlator for narrow frequency bands.

Fig. 13 Cross-correlation of near field pressure in the frequency band $2,000<w / 2 \pi<3,000 \mathrm{cps}$. $M_{\text {exit }}=.6, D=1, \mathrm{D} / \mathrm{D}=1, \theta=18^{\circ}$.

Fig. 14 Growth and decay of maxima of space-time correlations of frequency bands of near field pressure for several frequency bands and Machnumbers $\mathrm{X} / \mathrm{D}=1$, $\mathrm{D}=1$ in, $\theta=18^{\circ}$. 
Fig. 15 Crude breakdown of emitter types into sources, lateral dipoles and later quadrupoles, obtained from near field pressure correlations around the jet.

Fig. 16 Near field pressure power spectral density at several distances from nozzle exit plane.

Fig. 17 Far field pressure power spectral densities at $\mathrm{M}_{\text {exit }}=.8, \mathrm{D}=\frac{1}{2}$ in, $\mathrm{r}=36 \mathrm{D}$.

Fig. 18 Contours of constant root mean square pressures in far field. $M=.8$ and $.9, D=1 "$. Jet axis at $\theta=0^{\circ}$.

Fig. 19 Contours of constant rms pressure in far field for low frequencies only $f=w / 2 \pi$.

Fig. 20 Contours of constant rms pressure in far field for high frequencies only.

Fig. 2la Space time correlations between different freFig. 21b quency bands of near field pressure fluctuations. $\mathrm{M}=.6, \mathrm{D}=1$ in, $\mathrm{X} / \mathrm{D}=1$.

(I) Frequency band in kc of upstream microphone output.

(2) Frequency band in $\mathrm{kc}$ of downstream microphone output.

Fig. 22 Spectra of $u^{\prime}$ fluctuations in a free shear layer at one half instability wavelength from the start of the layer. Frequency in cps.

Fig. 23 Shadowgraph of the early shear layer in a 1 in diameter jet.

Fig. 24 Space-time correlations of near field pressures near a jet when the free shear layer is just above critical Reynolds number for instability. 


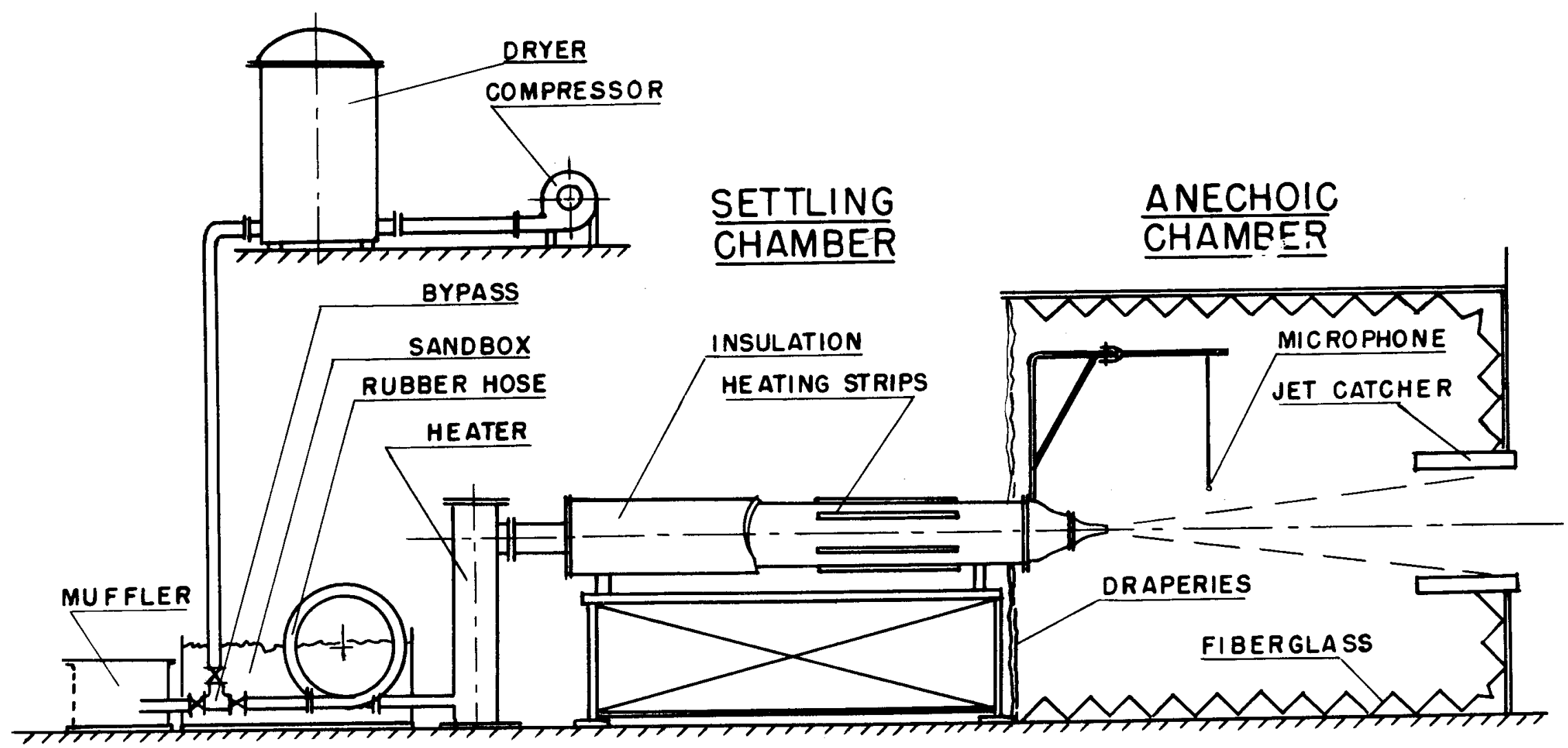

Fig. 1 Experimental arrangements. 


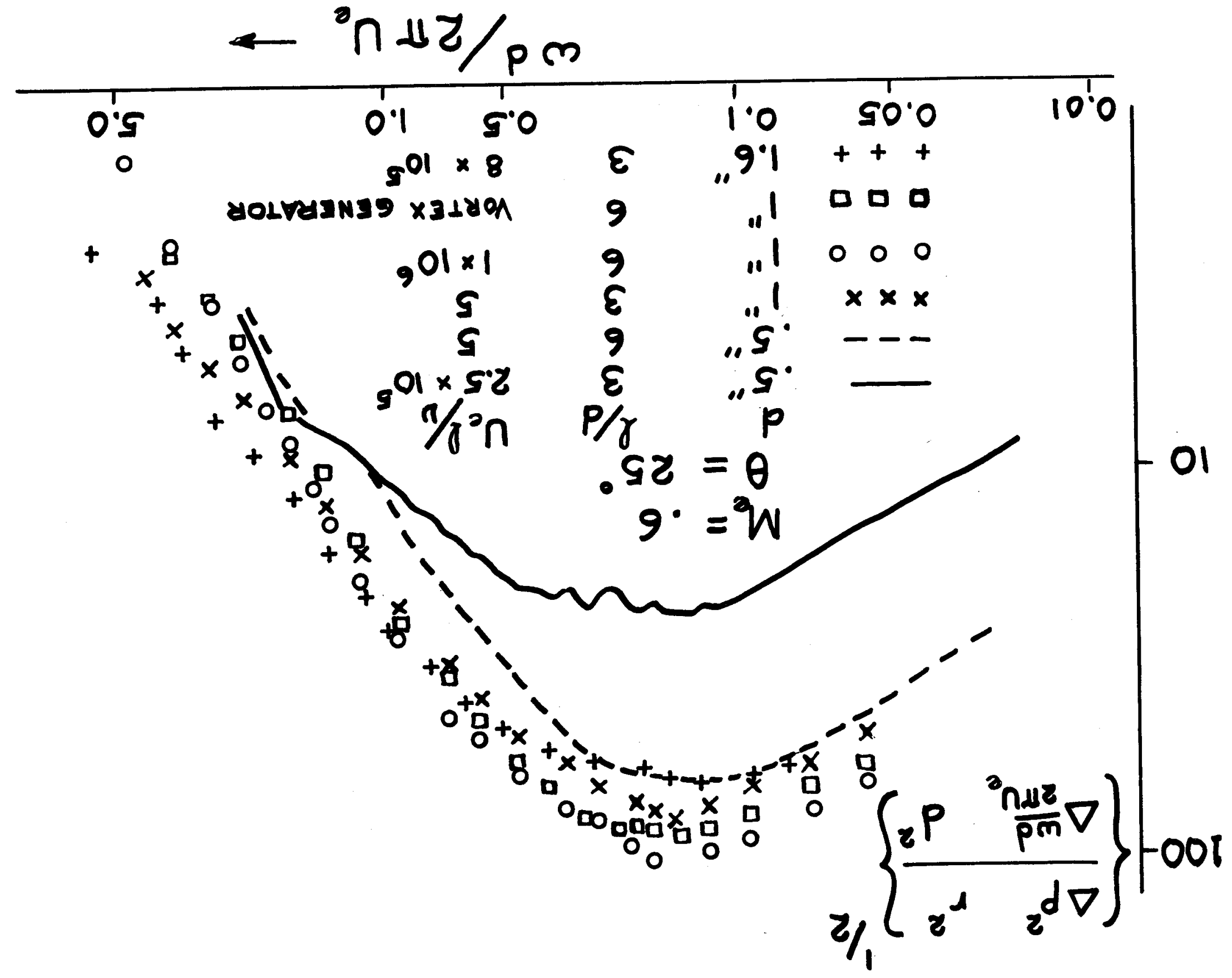



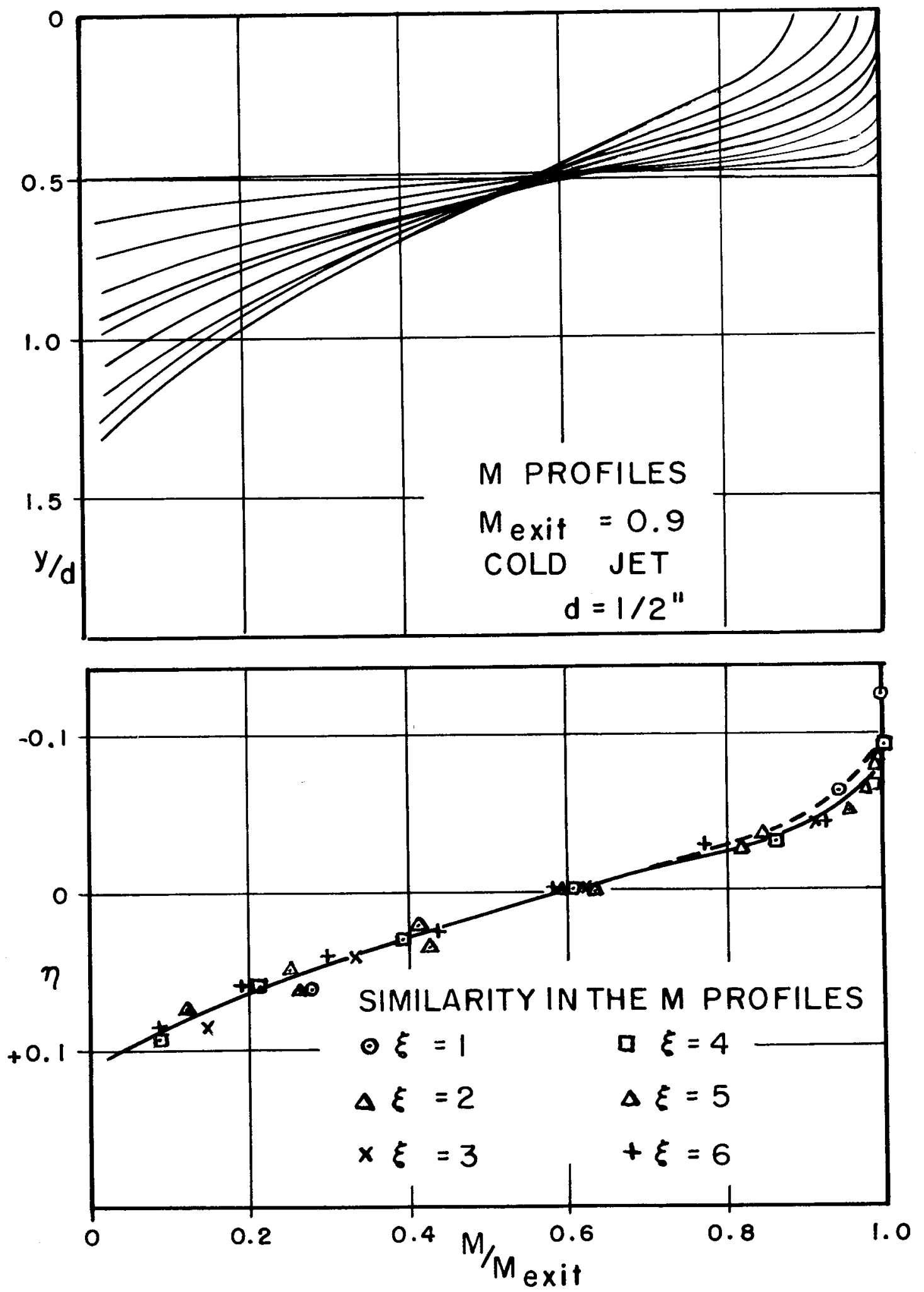

Fig. 3 Machnumber profiles for jet from $\frac{1}{2}$ in diameter nozzle. 


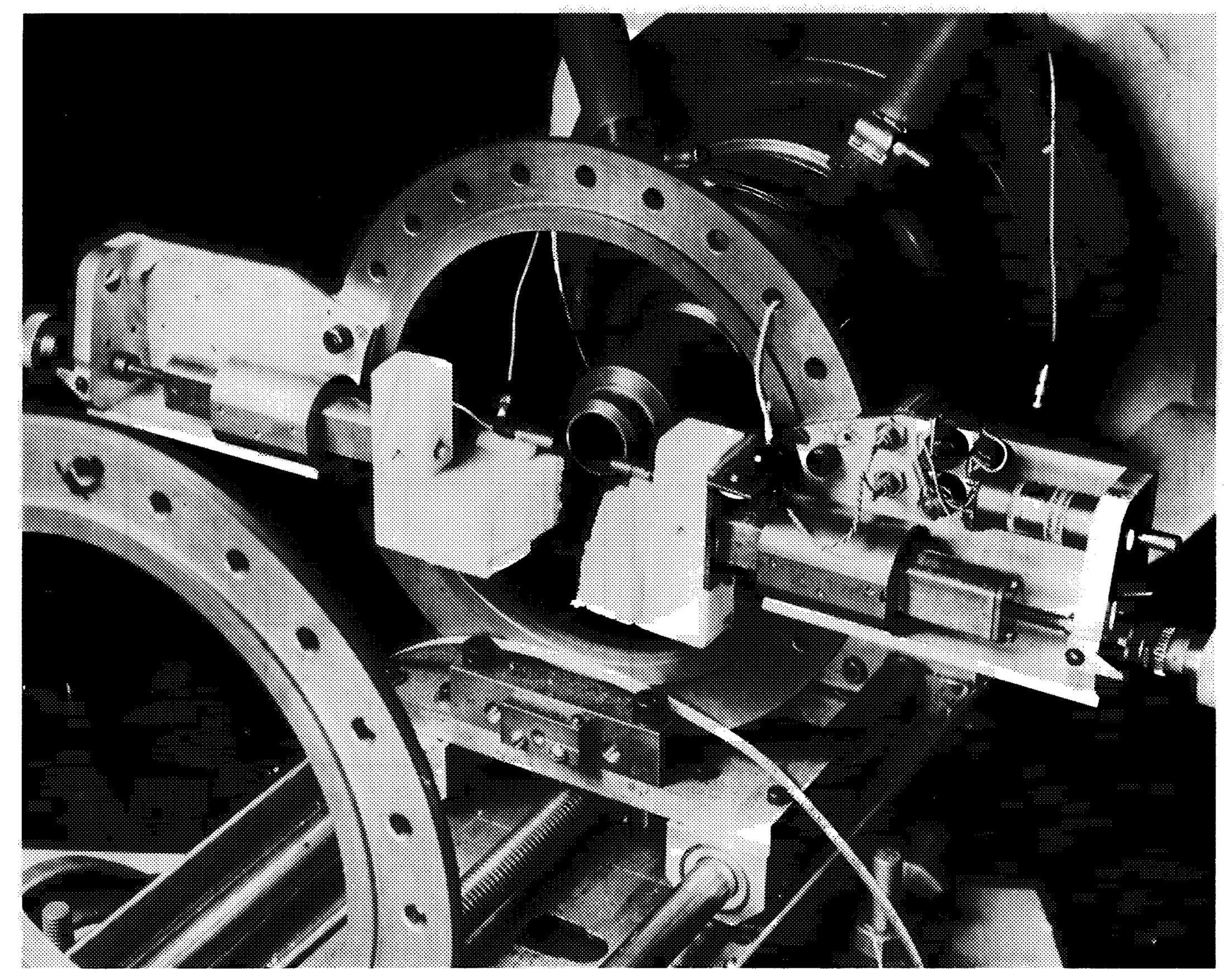

Fig. 4 Installation of microphones near a jet. 


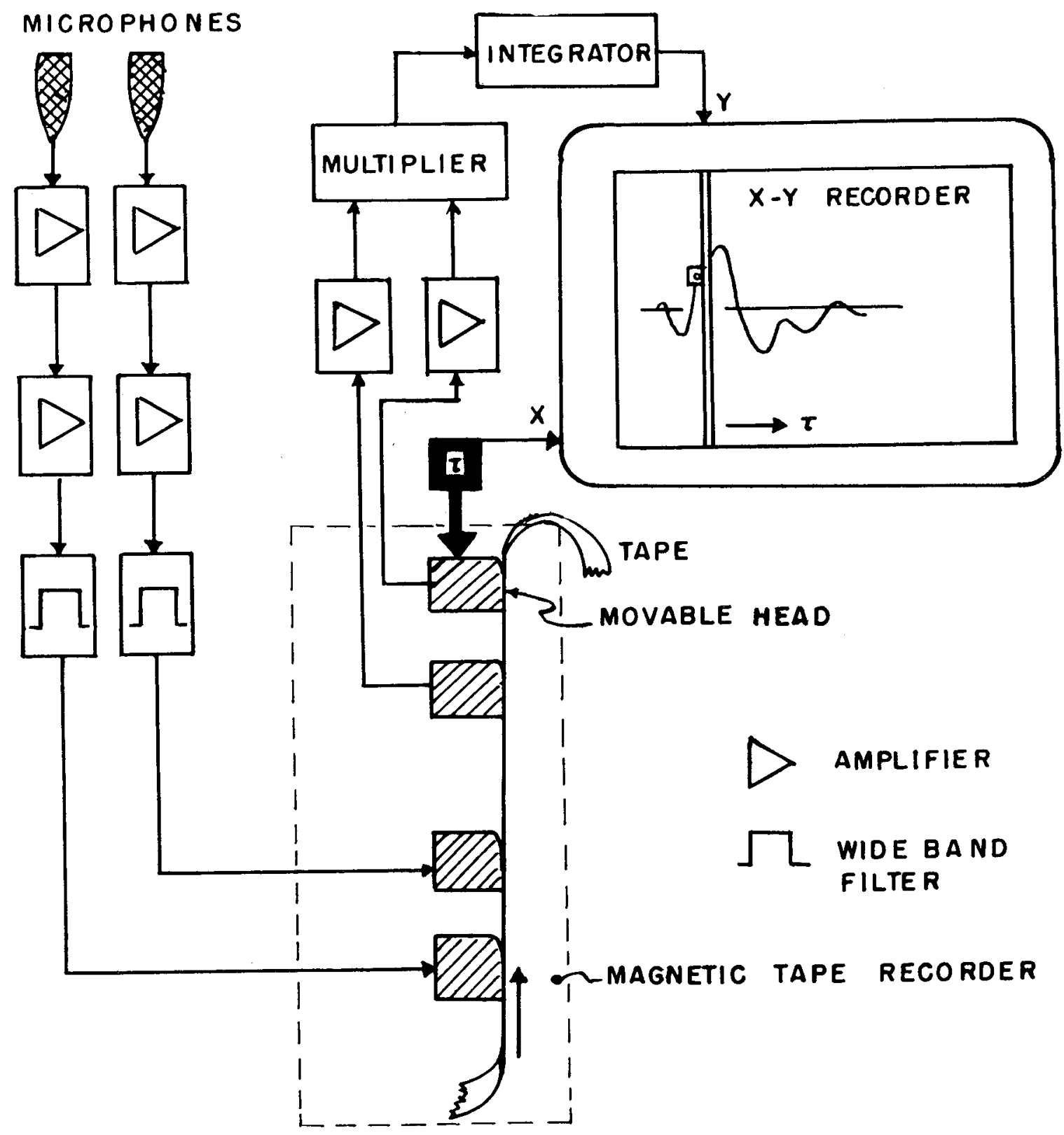

Fig. 5 Błock diagram of data processing system for obtaining space-time correlations. 


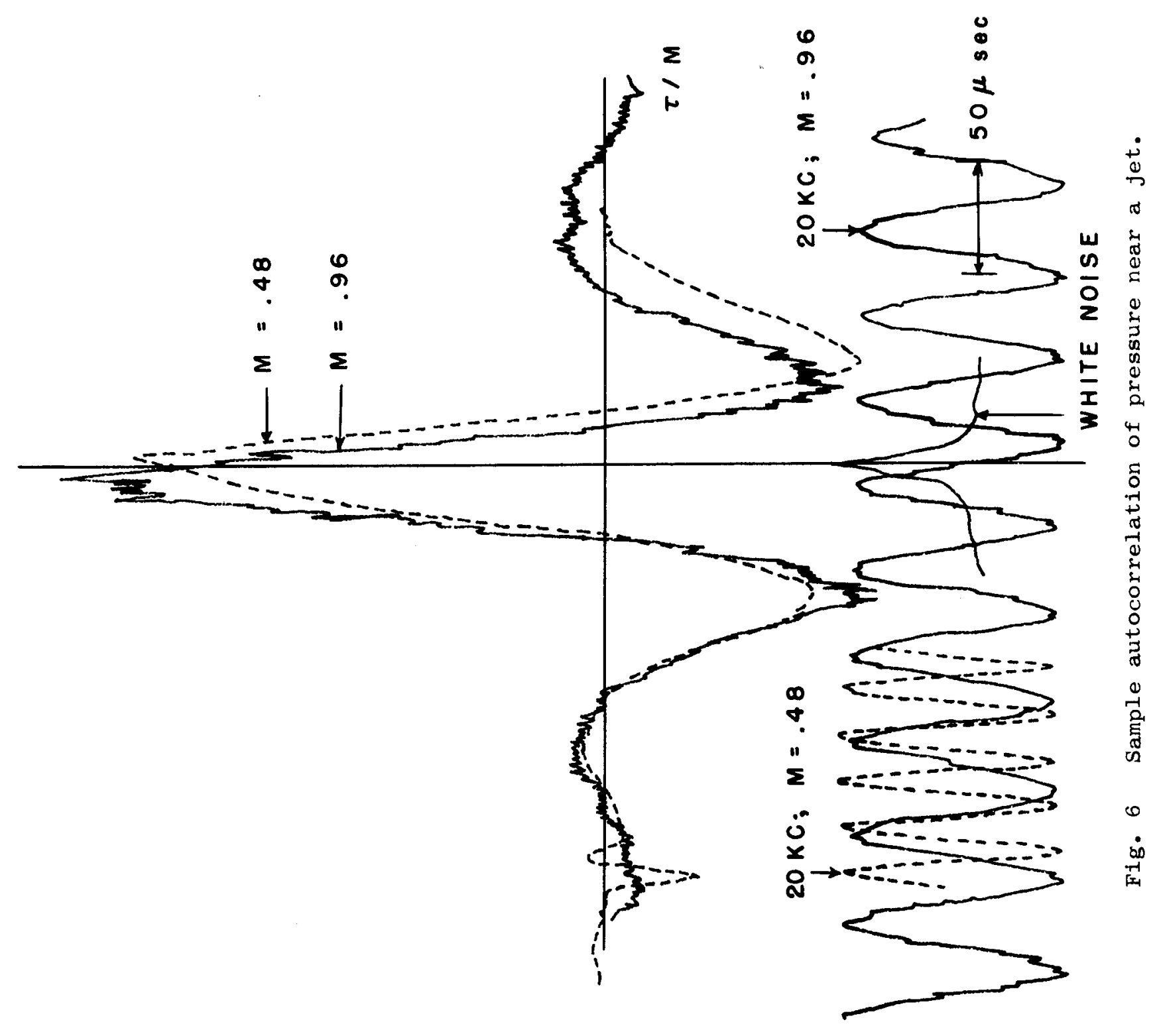




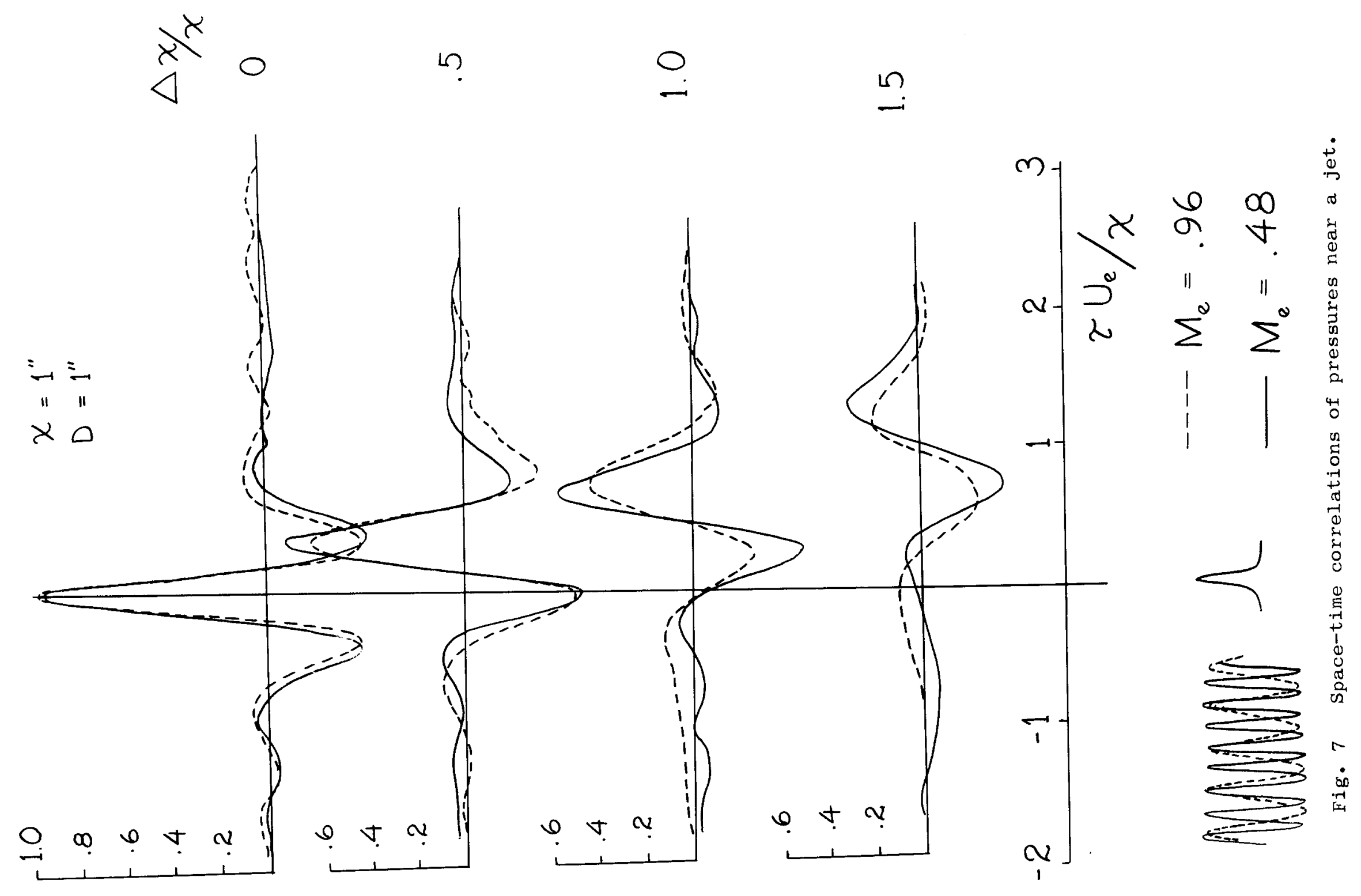




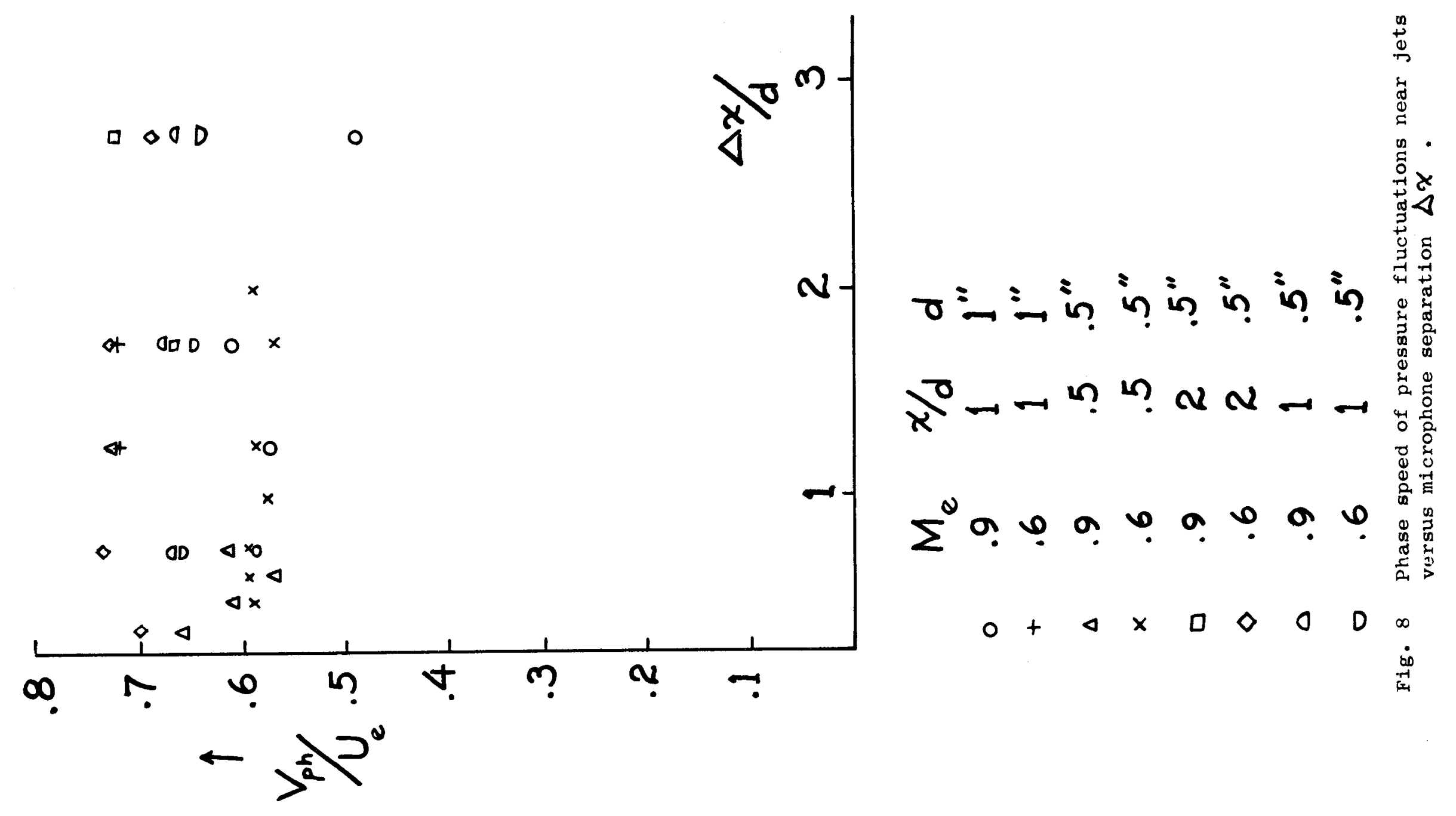




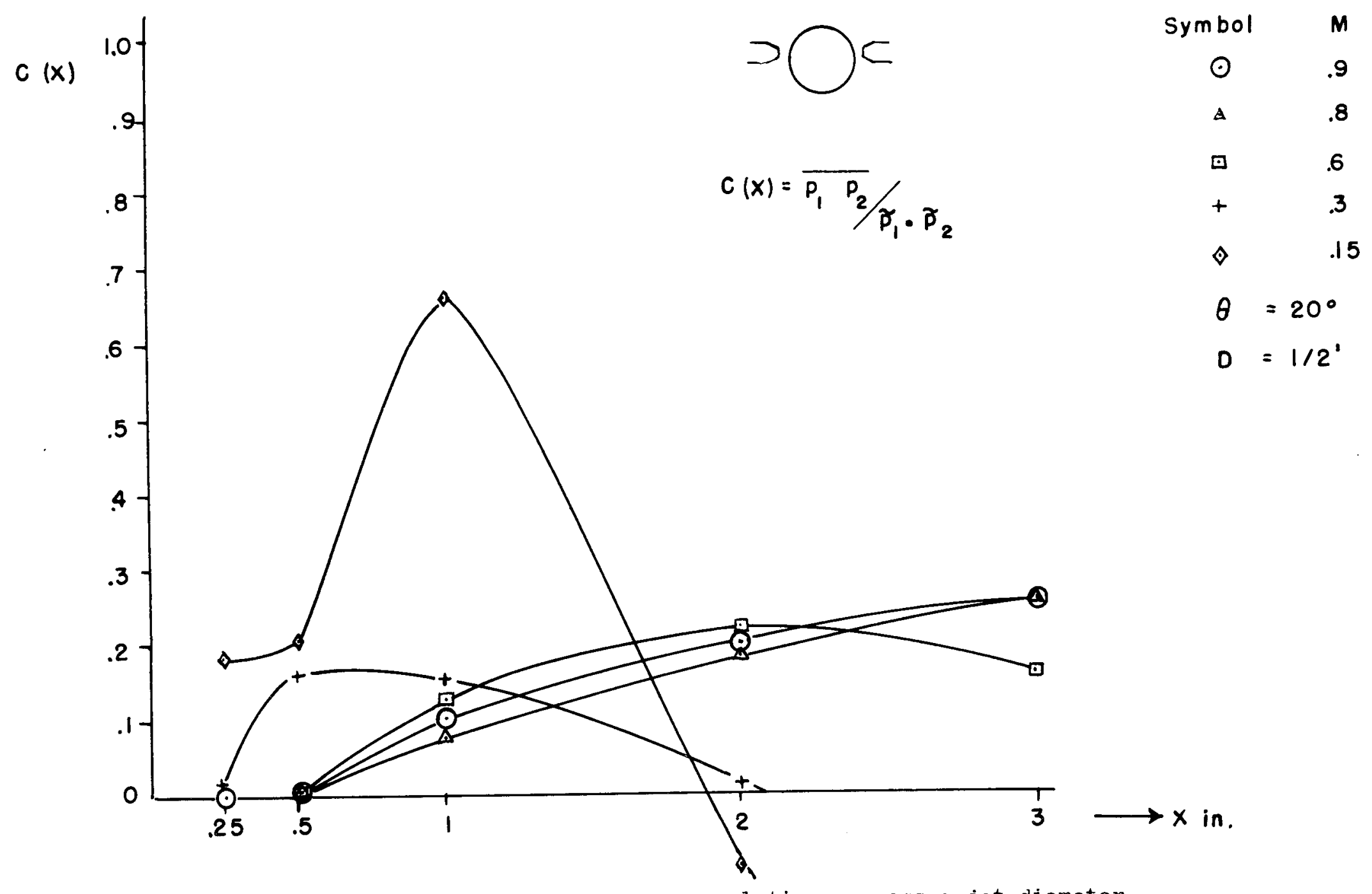

Fig. 9 Pressure correlations across a jet diameter versus distance from exit. 


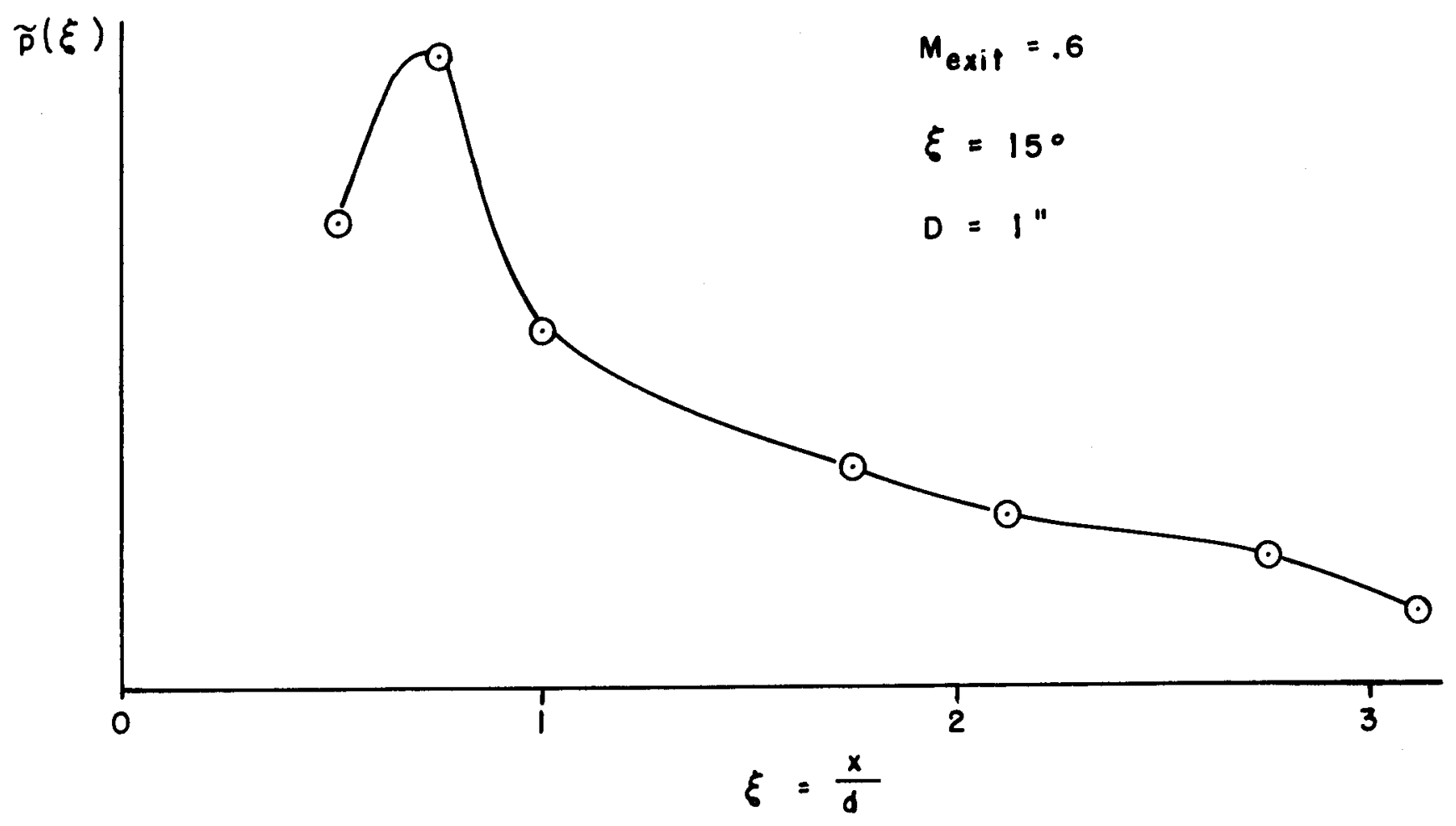

Fig. 10 Variation of rms pressure fluctuation with downstream distance. 


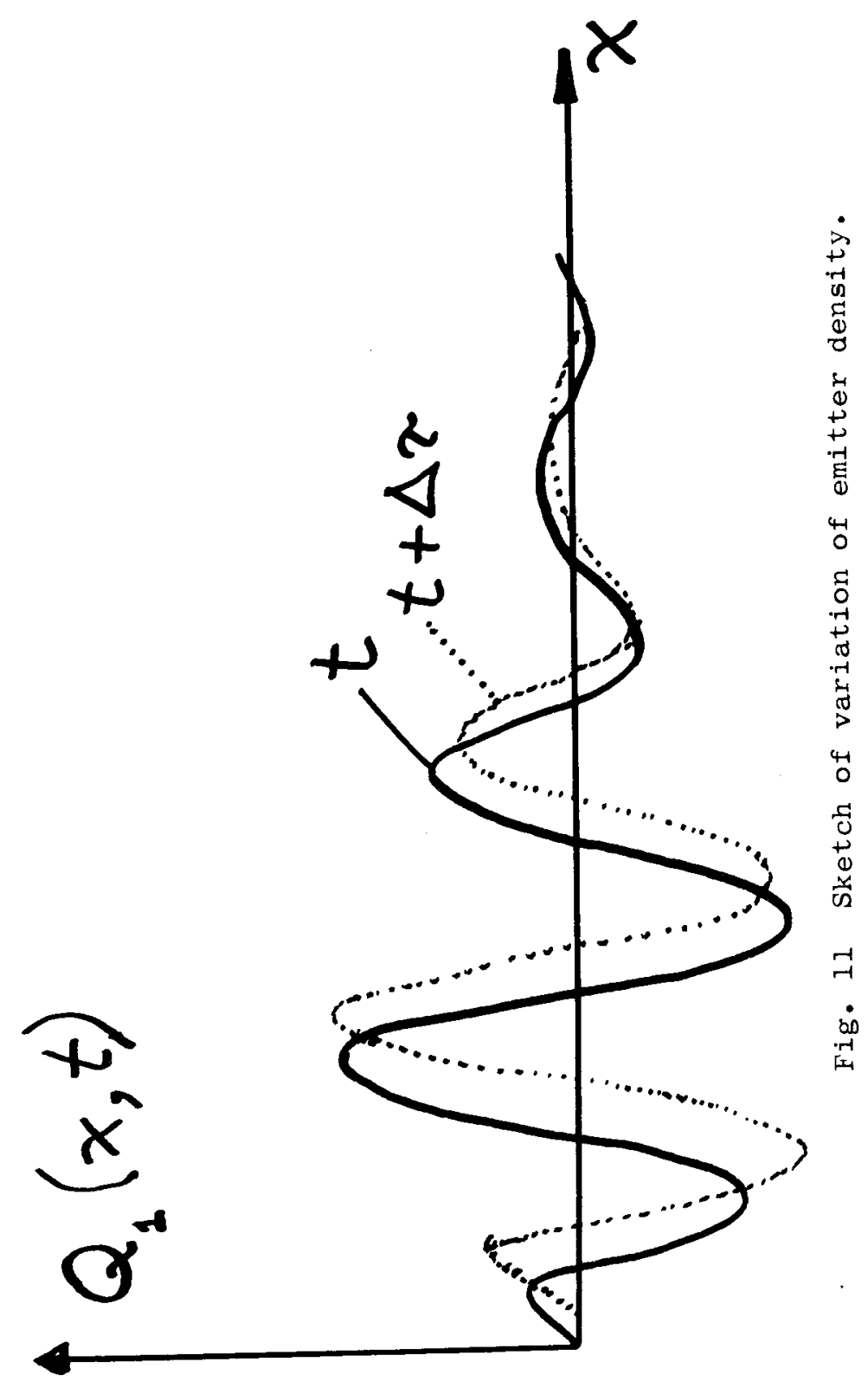




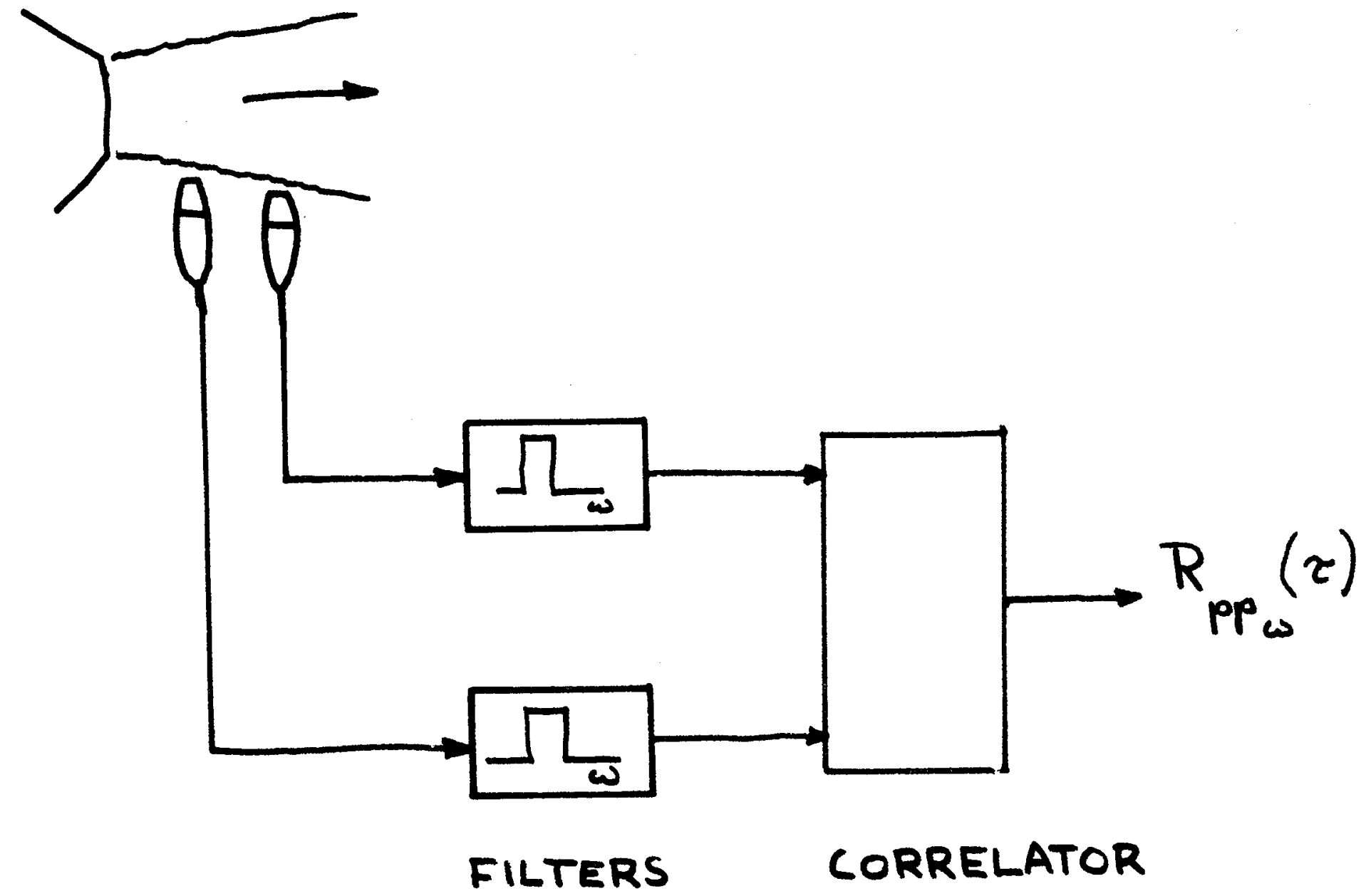

Fig. 12 Block diagram of cross correlator for narrow frequency bands. 


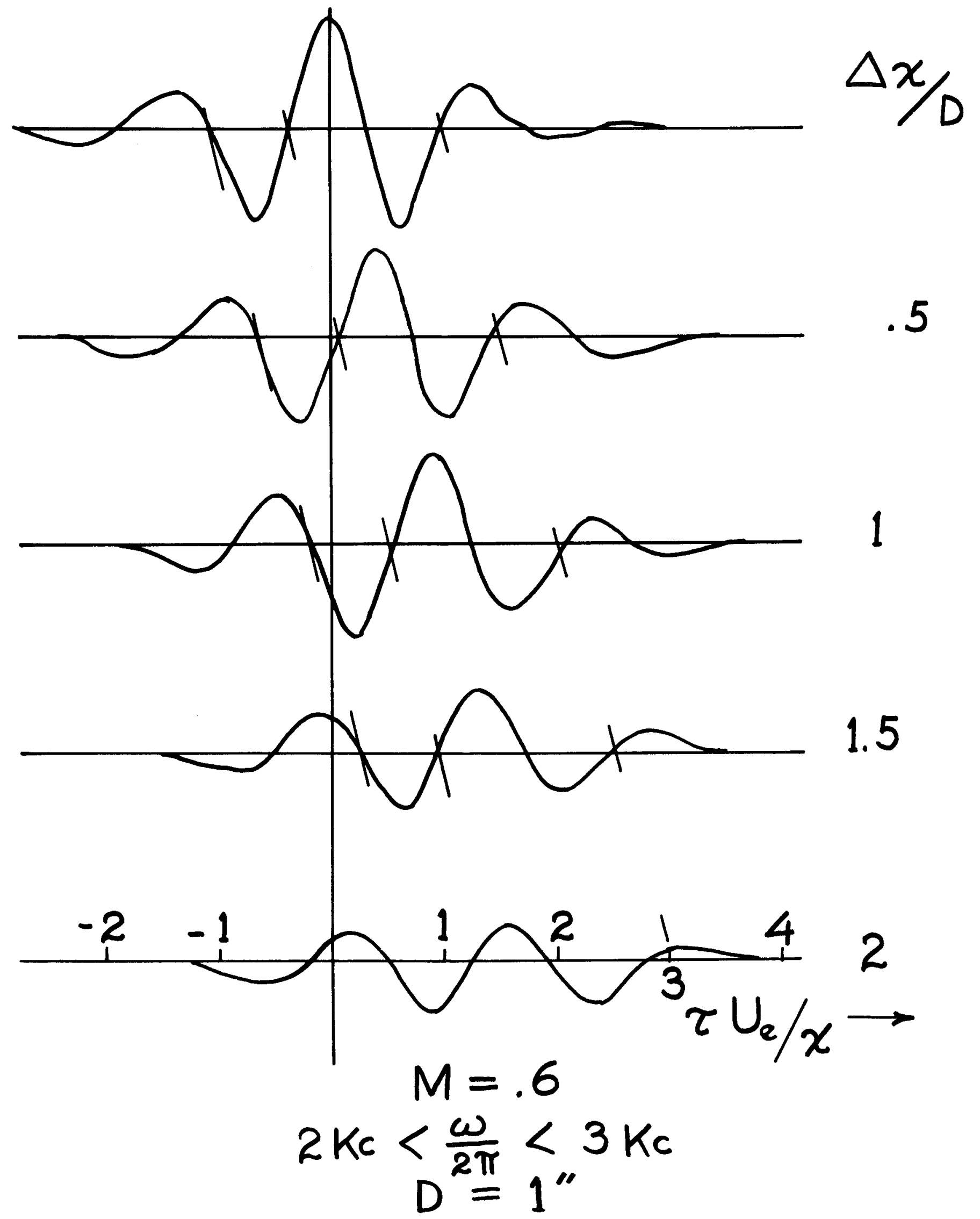

Fig. 13 Cross-correlation of near field pressure in the frequency band $2,000<\mathrm{w} / 2 \pi<3,000 \mathrm{cp}$
$\mathrm{M}_{\text {exit }}=.6, \mathrm{D}=1, \mathrm{X} / \mathrm{D}=1, \theta=18^{\circ}$ 


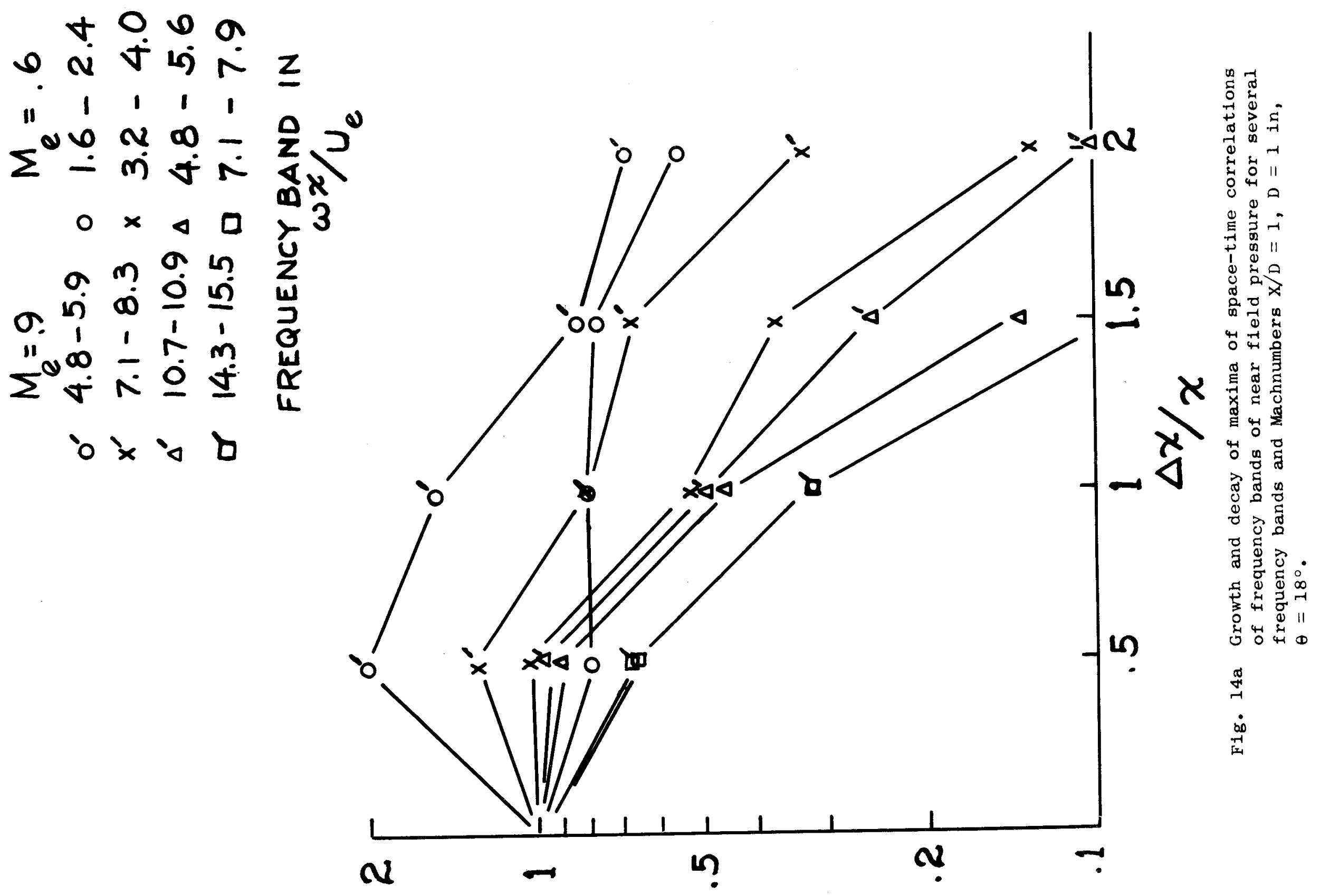


'ut $\mathrm{I}=\mathrm{a}^{\prime} \mathrm{L}=\mathrm{d} / \mathrm{X}$ sxәqumuqoen pure spueq אouәnbəxJ

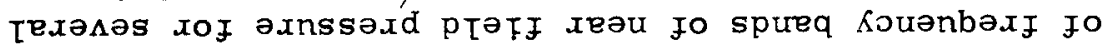

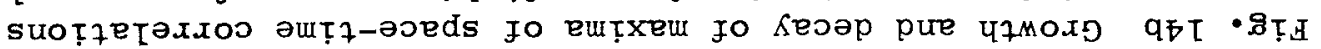

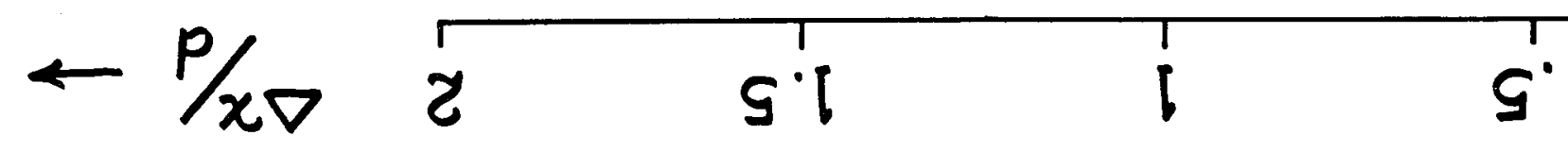

$Z 6 \cdot L>">\rightarrow 1 \cdot L \quad \circ$ $\varepsilon g \cdot \Omega>\|>S L+$ $96^{\circ} \varepsilon>\|>11^{\prime} \varepsilon \quad x$ $8 \varepsilon \cdot \zeta>">89^{\prime} \cdot$

$$
9 \cdot=\mathrm{W}
$$

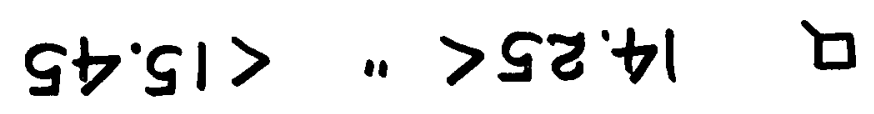
$98^{\circ}$ 이 $>$ " $>$ LO $\triangle$ $\varepsilon \varepsilon^{\prime} 8>\partial^{\prime \prime}>L 1 \cdot 2 \quad \square$ $b 6^{\circ} S>\frac{n}{\bar{x} M}>9 L^{\circ} b \quad 0$

$$
6^{\circ}=\mathrm{W}
$$

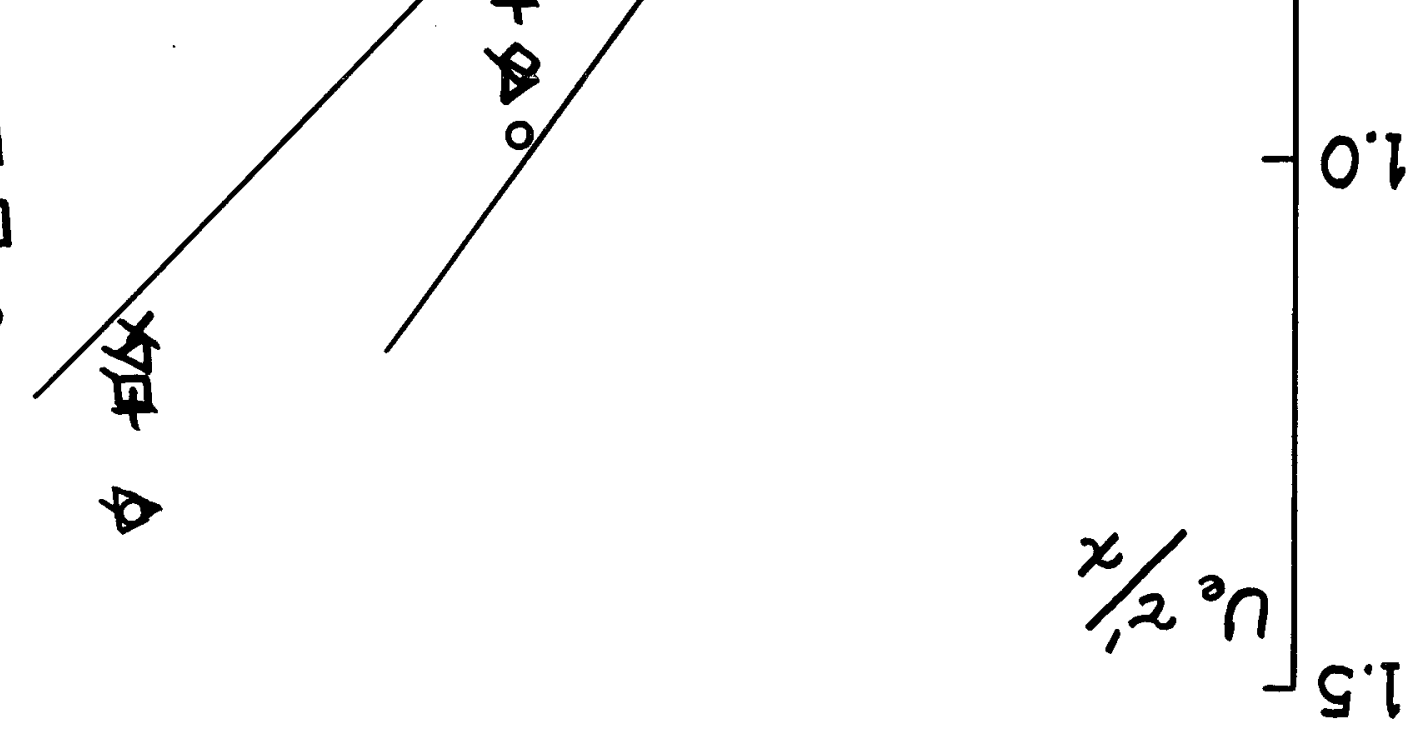


$\bigcap^{\alpha} c_{p p}(x, \alpha)=$

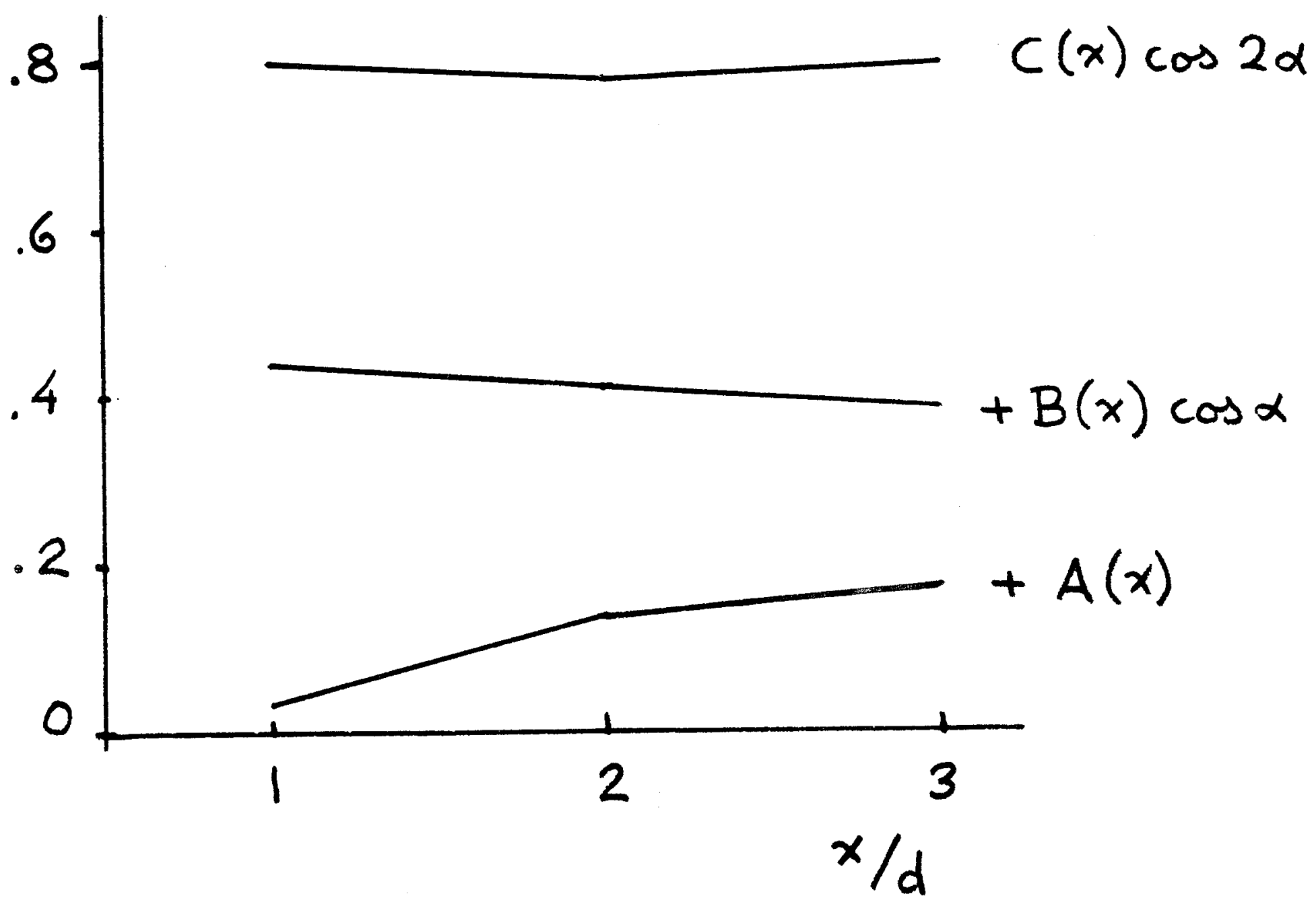

Fig. 15 Crude breakdown of emitter types into sources, lateral
dipoles and later quadropoles, obtained from near field pressure correlations around the jet. 


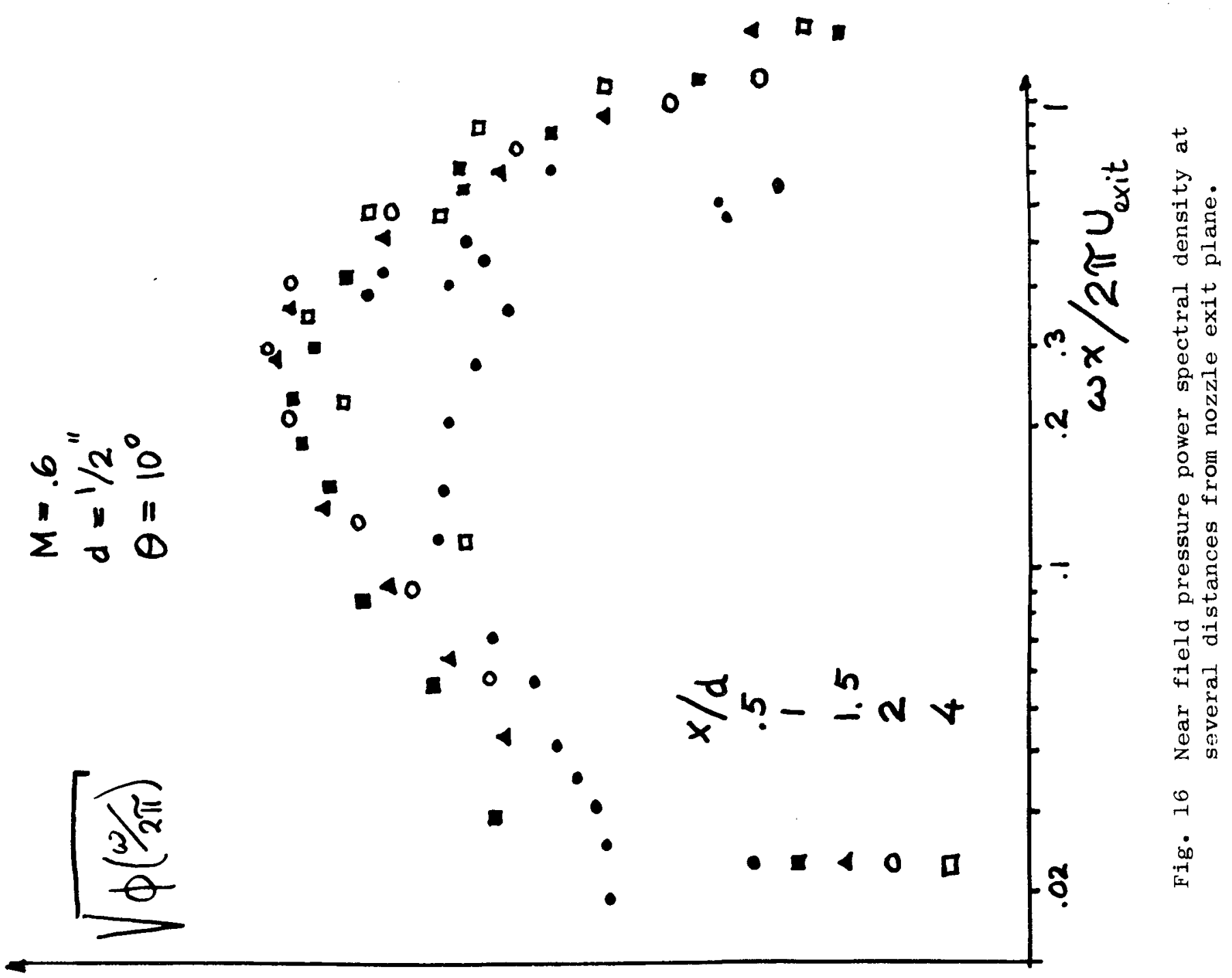




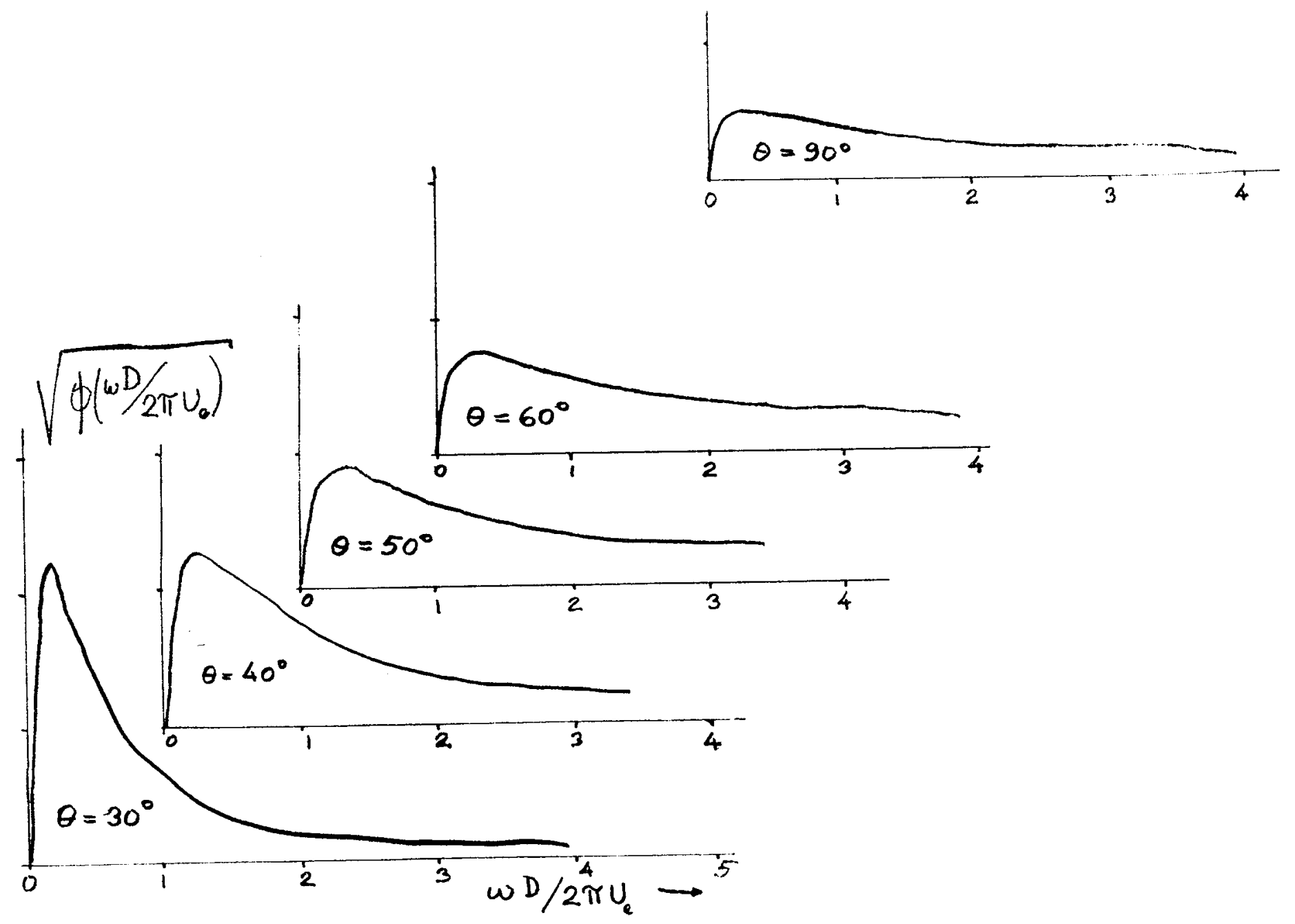

Fig. 17 Far field pressure power spectral densities at $\mathrm{M}_{\text {Exit }}=.8, \mathrm{D}=\frac{1}{2}$ in, $\mathrm{r}=36 \mathrm{D}$. 


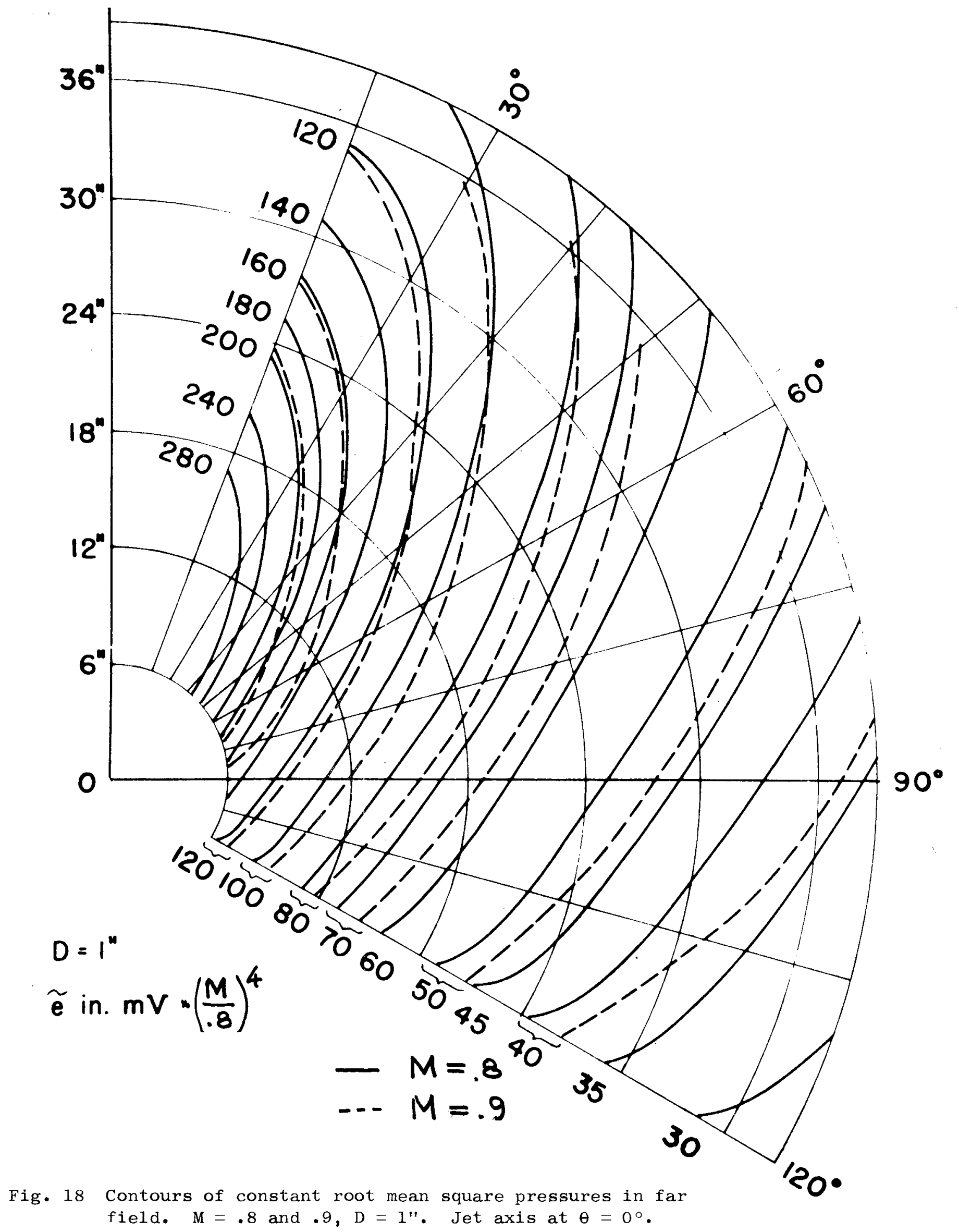




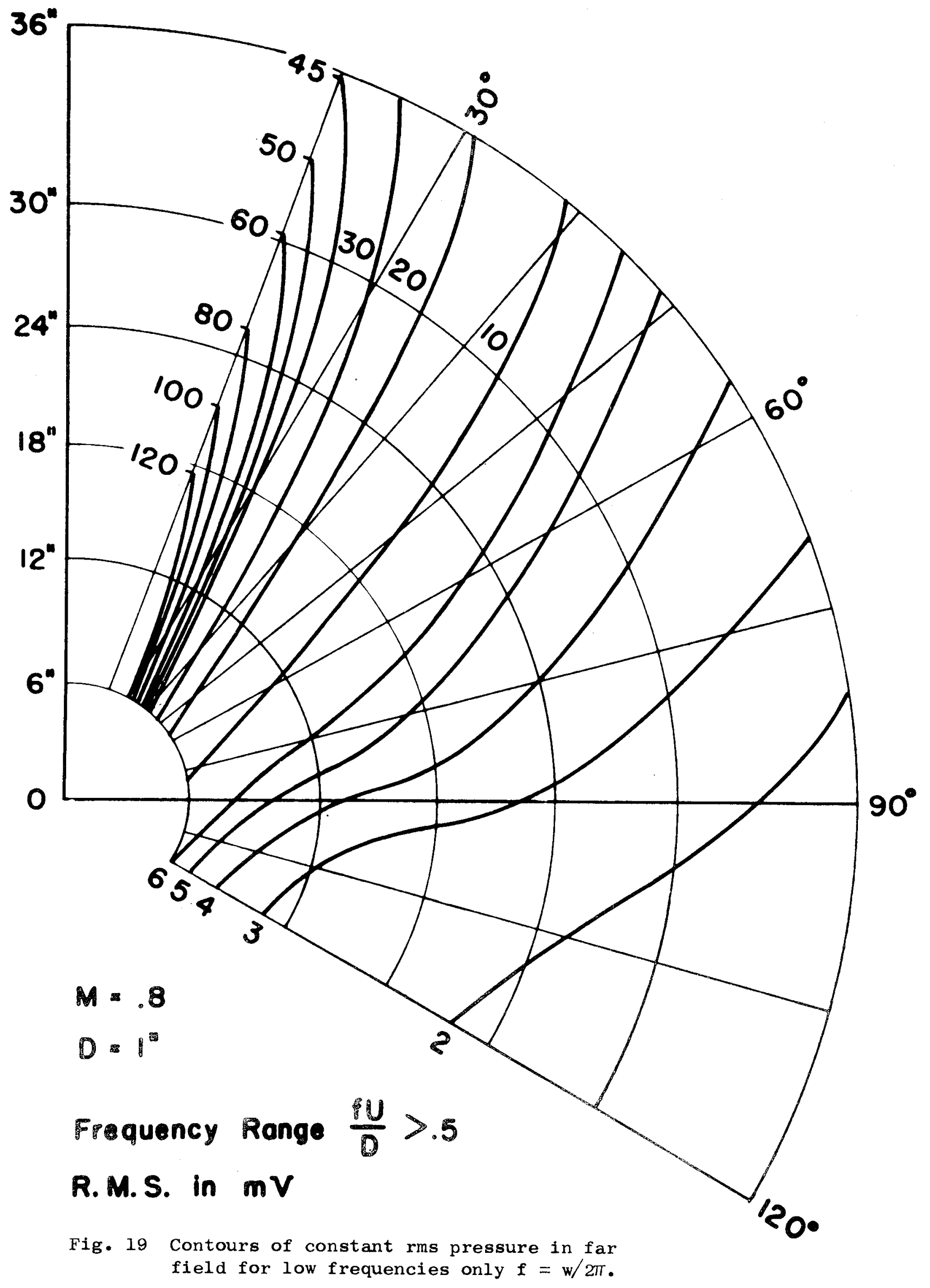




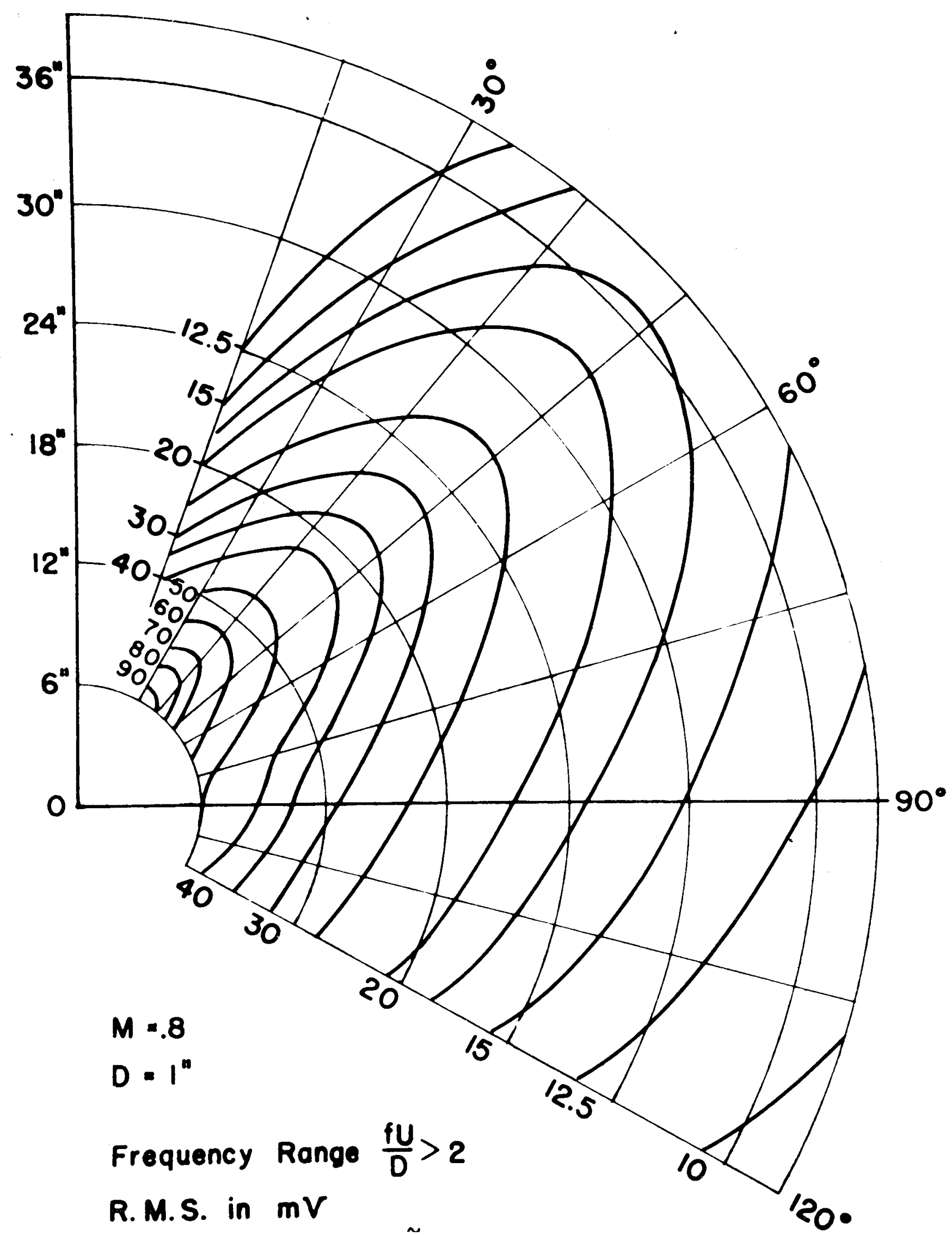

Fig. 20 Contours of constant rms pressure in far field for high frequencies only. 

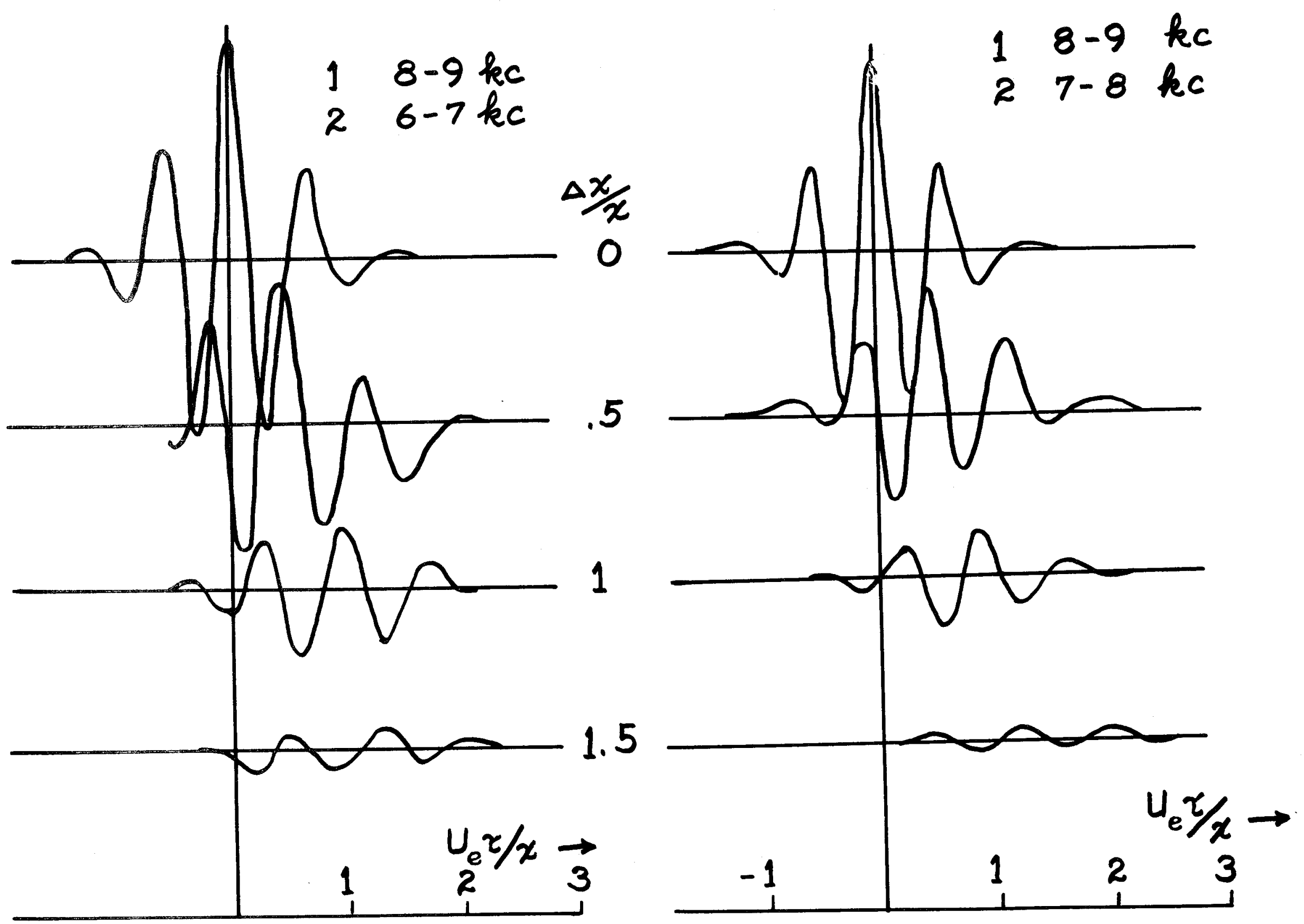

Fig. Ila Space time correlations between different frequency bands of near field pressure fluctuations. $M=.6, D=1$ in, $\mathrm{X} / \mathrm{D}=1$.

(1) Frequency band in kc of upstream microphone output.

(2) Frequency band in $\mathrm{kc}$ of downstream microphone output. 

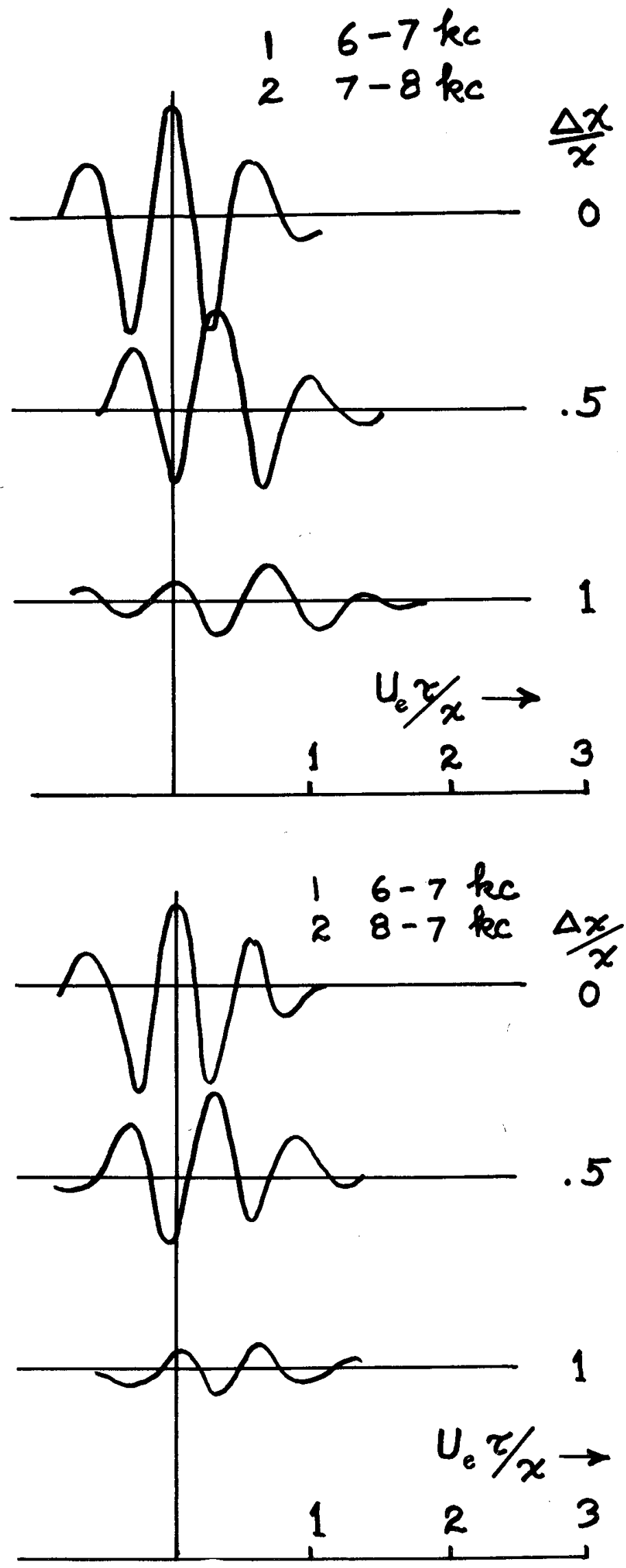

Fig. 21b Space time correlations between different frequency bands of near field pressure fluctuations. $\mathrm{M}=.6, \mathrm{D}=1 \mathrm{in}, \mathrm{X} / \mathrm{D}=1$.

(1) Frequency band in kc of upstream microphone output.

(2) Frequency band in kc of downstream microphone output. 


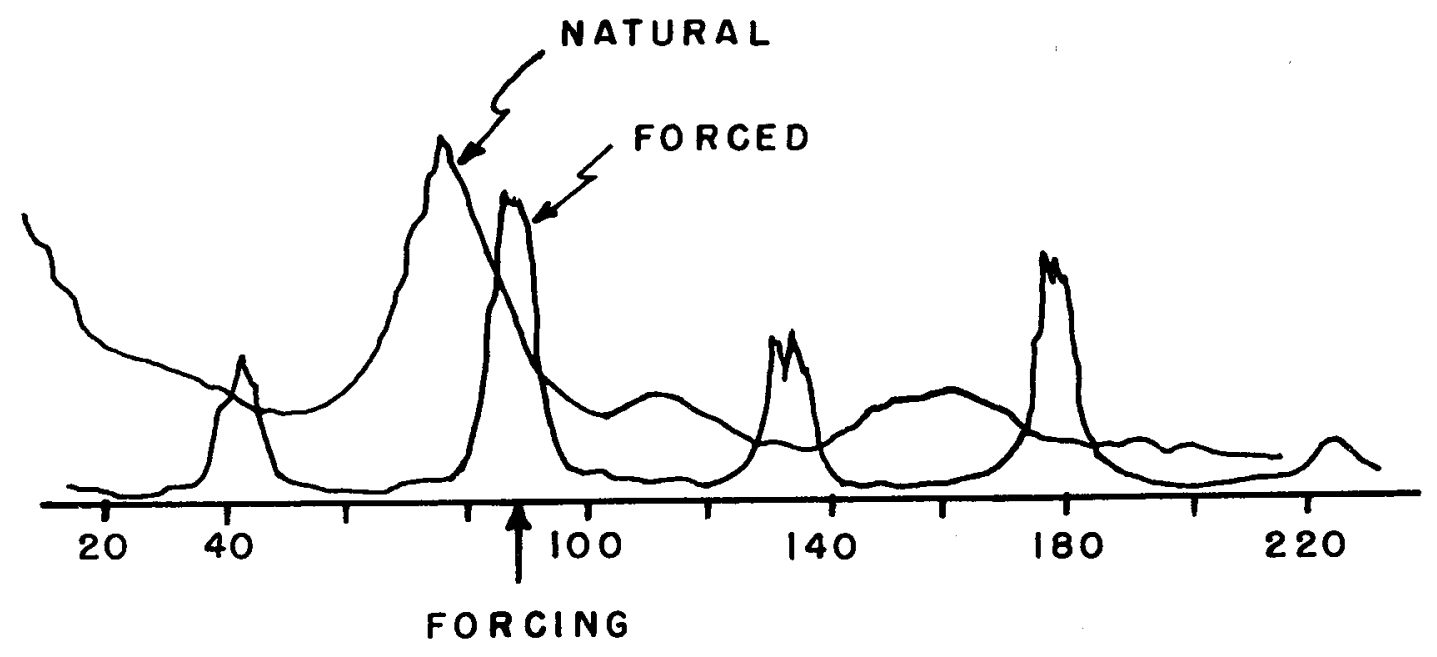

FREQ.

Fig. 22 Spectra of $u^{\prime}$ fluctuations in a free shear layer at one half instability wavelength from the start of the layer. Frequency in cps. 


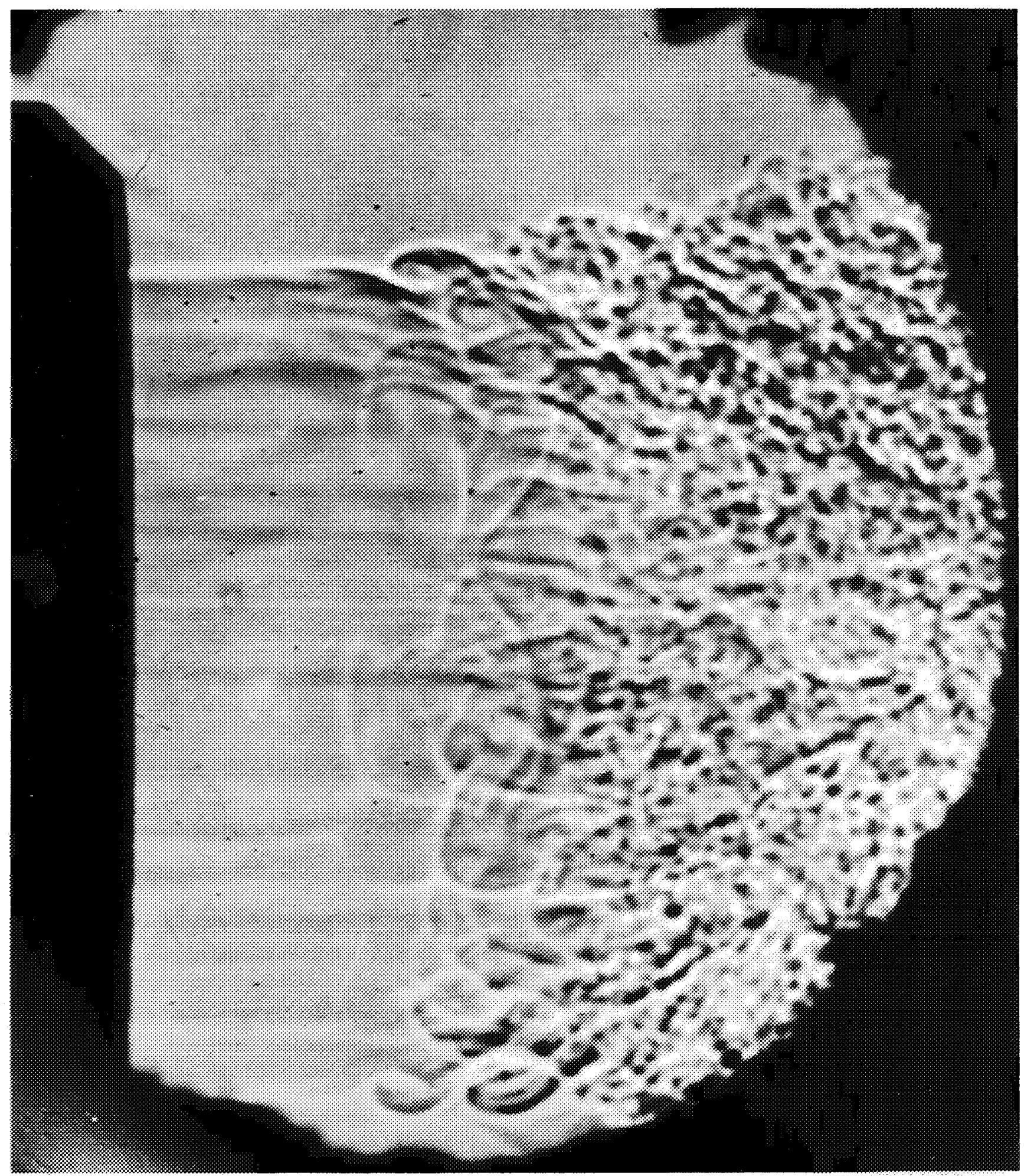

Fig. 23 Shadowgraph of the early shear layer in a 1 in diameter jet. 


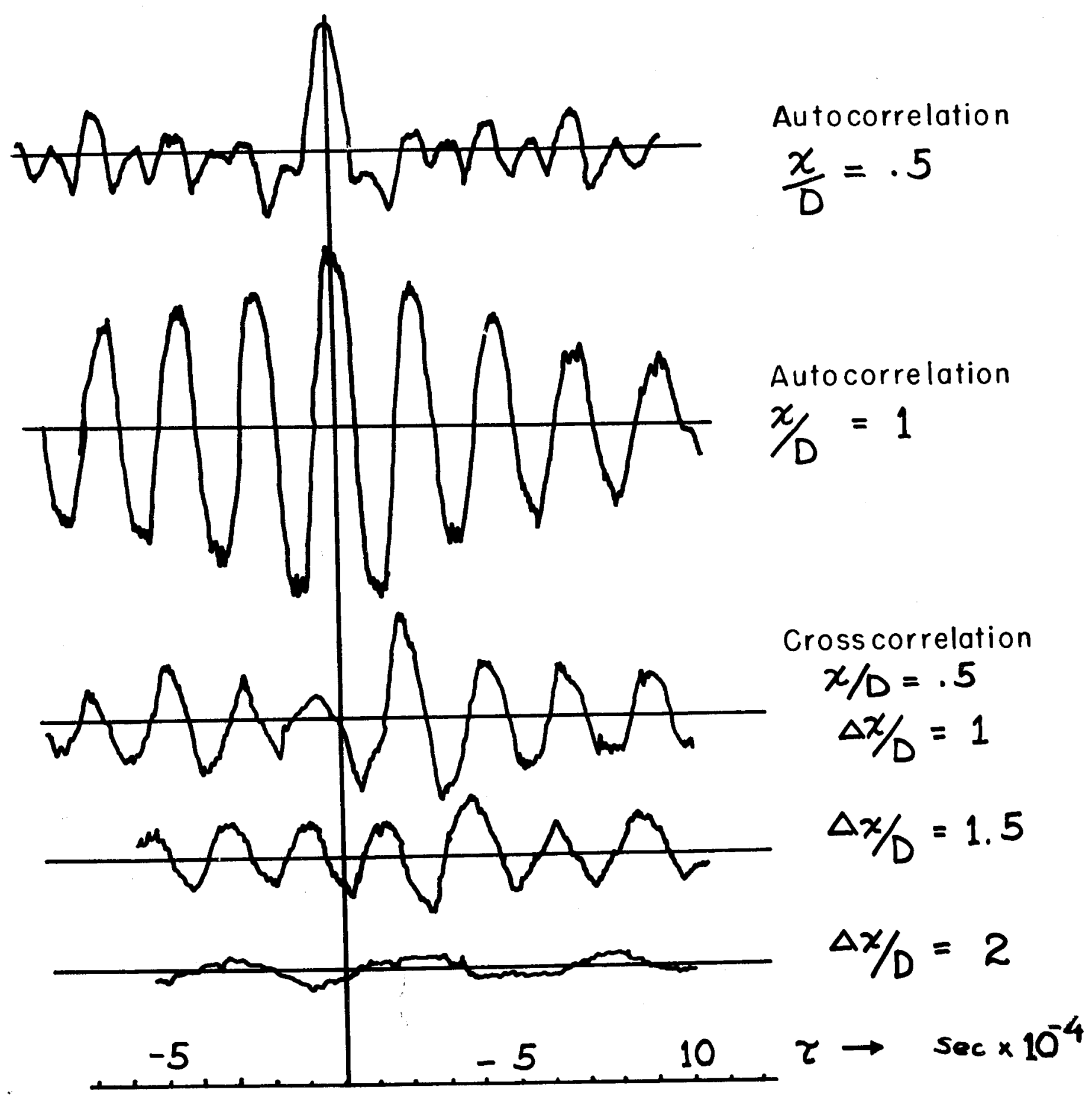

Fig. 24 Space-time correlations of near field pressures near a jet when the free shear layer is just above critical Reynolds number for instability. 